\title{
NONLINEAR EQUATIONS WITH GRADIENT NATURAL GROWTH AND DISTRIBUTIONAL DATA, WITH APPLICATIONS TO A SCHRÖDINGER TYPE EQUATION
}

\author{
KARTHIK ADIMURTHI ${ }^{1}$ AND NGUYEN CONG PHUC ${ }^{2}$
}

\begin{abstract}
We obtain necessary and sufficient conditions with sharp constants on the distribution $\sigma$ for the existence of a globally finite energy solution to the quasilinear equation with a gradient source term of natural growth of the form $-\Delta_{p} u=|\nabla u|^{p}+\sigma$ in a bounded open set $\Omega \subset \mathbb{R}^{n}$. Here $\Delta_{p}, p>1$, is the standard $p$-Laplacian operator defined by $\Delta_{p} u=\operatorname{div}\left(|\nabla u|^{p-2} \nabla u\right)$. The class of solutions that we are interested in consists of functions $u \in W_{0}^{1, p}(\Omega)$ such that $e^{\mu u} \in W_{0}^{1, p}(\Omega)$ for some $\mu>0$ and the inequality

$$
\int_{\Omega}|\varphi|^{p}|\nabla u|^{p} d x \leq A \int_{\Omega}|\nabla \varphi|^{p} d x
$$

holds for all $\varphi \in C_{c}^{\infty}(\Omega)$ with some constant $A>0$. This is a natural class of solutions at least when the distribution $\sigma$ is nonnegative. The study of $-\Delta_{p} u=|\nabla u|^{p}+\sigma$ is applied to show the existence of globally finite energy solutions to the quasilinear equation of Schrödinger type $-\Delta_{p} v=\sigma v^{p-1}, v \geq 0$ in $\Omega$, and $v=1$ on $\partial \Omega$, via the exponential transformation $u \mapsto v=e^{\frac{u}{p-1}}$.
\end{abstract}

\section{INTRODUCTION}

The main goal of this paper is to address the solvability of quasilinear elliptic equations with gradient nonlinearity of natural growth of the form

$$
\begin{cases}-\Delta_{p} u=|\nabla u|^{p}+\sigma & \text { in } \Omega \\ u=0 & \text { on } \partial \Omega\end{cases}
$$

in a bounded open set $\Omega \subset \mathbb{R}^{n}$. Here $\Delta_{p} u:=\operatorname{div}\left(|\nabla u|^{p-2} \nabla u\right), p>1$, is the $p$-Laplacian and the datum $\sigma$ is a distribution in $\Omega$. More generally, we also consider the equation

$$
\begin{cases}-\operatorname{div} \mathcal{A}(x, u, \nabla u)=\mathcal{B}(x, u, \nabla u)+\sigma & \text { in } \quad \Omega \\ u=0 & \text { on } \partial \Omega\end{cases}
$$

\footnotetext{
${ }^{1}$ Supported in part by National Research Foundation of Korea grant funded by the Korean government (MEST) (NRF-2015R1A2A1A15053024).

${ }^{2}$ Supported in part by Simons Foundation, award number 426071.
} 
where the principal operator $\operatorname{div} \mathcal{A}(x, u, \nabla u)$ is a Leray-Lions operator defined on $W_{0}^{1, p}(\Omega)$ and $|\mathcal{B}(x, u, \nabla u)| \lesssim|\nabla u|^{p}$.

The precise assumptions on the nonlinearities $\mathcal{A}, B$ and the the precise definition of solutions to (1.2) will be given in Section 2. Here we emphasize that in this paper we are interested only in finite energy solutions $u$ with zero boundary condition in the sense that $u \in W_{0}^{1, p}(\Omega)$. The energy space $W_{0}^{1, p}(\Omega)$ is defined as the completion of $C_{c}^{\infty}(\Omega)$ under the semi-norm $\|\nabla(\cdot)\|_{L^{p}(\Omega)}$.

As an application of the study of (1.1), we also obtain existence of finite energy solution to the quasilinear Schrödinger type equation

$$
-\Delta_{p} v=(p-1)^{1-p} \sigma v^{p-1} \text { in } \Omega, \quad v \geq 0 \text { in } \Omega, \quad v=1 \text { on } \partial \Omega .
$$

Equation (1.1) is a prototype for quasilinear equations with natural growth in the gradient that has attracted a lot of attention in the past years. It can be viewed as a quasilinear stationary version of a time-dependent viscous Hamilton-Jacobi equation, also known as the Kardar-Parisi-Zhang equation, which appears in the physical theory of surface growth $[23,24]$.

As far as existence is concerned, the nonlinearity $|\nabla u|^{p}$ in (1.1) is considered "to have the bad sign" and by now it is well-known that in order for (1.1) to have a solution the datum $\sigma$ must be both small and regular enough. In particular, if $\sigma$ is a nonnegative distribution in $\Omega$ (i.e., a nonnegative locally finite measure in $\Omega$ ), then a necessary condition for the first equation in $(1.1)$ to have a $W_{\text {loc }}^{1, p}(\Omega)$ solution is that (see $\left.[20,21,22]\right)$

$$
\int_{\Omega}|\varphi|^{p} d \sigma \leq \lambda \int_{\Omega}|\nabla \varphi|^{p} d x \quad \text { for all } \varphi \in C_{c}^{\infty}(\Omega)
$$

with $\lambda=(p-1)^{p-1}$. Moreover, when $\sigma \geq 0$ the nonlinear term itself also obeys a similar Poincaré-Sobolev inequality

$$
\int_{\Omega}|\varphi|^{p}|\nabla u|^{p} d x \leq A \int_{\Omega}|\nabla \varphi|^{p} d x \quad \text { for all } \varphi \in C_{c}^{\infty}(\Omega),
$$

with $A=p^{p}$.

Thus a natural space of solutions associated to (1.1) is the space $\mathcal{S}$ of functions $u \in$ $W_{0}^{1, p}(\Omega)$ such that (1.5) holds for some $A>0$. The main question we wish to address here is to find an optimal (largest) space $\mathcal{D}$ of 'data' so that whenever $\sigma \in \mathcal{D}$ with sufficiently small norm $\|\sigma\|_{\mathcal{D}}$ then (1.1) admits a solution in $\mathcal{S}$. In the case $\sigma \geq 0$ we can completely characterize the existence of finite energy solutions to (1.1) in the following theorem. We 
remark again that in this case all $W_{0}^{1, p}(\Omega)$ solutions automatically belong to $\mathcal{S}$ and $(1.5)$ holds with $A=p^{p}$.

Theorem 1.1. Let $\sigma$ be a nonnegative locally finite measure in $\Omega$. If (1.1) has a solution in $u \in W_{0}^{1, p}(\Omega)$ then $\sigma \in\left(W_{0}^{1, p}(\Omega)\right)^{*}$ and (1.4) holds with $\lambda=(p-1)^{p-1}$. Conversely, if $\sigma \geq 0, \sigma \in\left(W_{0}^{1, p}(\Omega)\right)^{*}$, and (1.4) holds with $0<\lambda<(p-1)^{p-1}$ then (1.1) has a nonnegative solution in $W_{0}^{1, p}(\Omega)$ such that $e^{\frac{\delta u}{p-1}}-1 \in W_{0}^{1, p}(\Omega)$ for all $\delta \in\left[0, \delta_{0}\right)$ where $\delta_{0}=(p-1) \lambda^{\frac{-1}{p-1}}$.

In the linear case, $p=2$, these necessary and sufficient conditions have been observed in [17]. See also [1] (for $p=2$ ) and [19] for certain related results that were obtained by different methods. We remark that, under a mild restriction on the domain, by Hardy's inequality (see $[3,25])$, Theorem 1.1 covers the case of unbounded measure such as $\sigma=\varepsilon \operatorname{dist}(x, \partial \Omega)^{-1}$ for some $\varepsilon>0$. It is also worth mentioning that in the case $p=2$ and $\sigma$ is a nonnegative locally finite measure, other sharp existence results for (1.1) were obtained in [20] for $\Omega=\mathbb{R}^{n}$ and recently in [17] for bounded domains $\Omega$ with $C^{2}$ boundary under a very weak notion of solution and boundary conditions.

The first part of Theorem 1.1 follows from the known necessary condition (1.4), Hölder's inequality, and the assumption that $\nabla u \in L^{p}(\Omega)$, since we have

$$
\sigma=-|\nabla u|^{p}-\operatorname{div}\left(|\nabla u|^{p-2} \nabla u\right) \leq-\operatorname{div}\left(|\nabla u|^{p-2} \nabla u\right) .
$$

On the other hand, the second part is a consequence of Theorem 1.2 below that treats even sign changing distribution datum $\sigma$. This in fact is the main result that will be obtained in this paper.

Theorem 1.2. (i) Suppose that (1.1) has a solution in $u \in W_{0}^{1, p}(\Omega)$ such that (1.5) holds for some $A>0$ then it necessarily holds that $\sigma=\operatorname{div}(F)-|F|^{\frac{p}{p-1}}$ for a vector field $F \in L^{\frac{p}{p-1}}\left(\Omega, \mathbb{R}^{n}\right)$ such that

$$
\int_{\Omega}|F|^{\frac{p}{p-1}}|\varphi|^{p} d x \leq A \int_{\Omega}|\nabla \varphi|^{p} d x \quad \text { for all } \varphi \in C_{c}^{\infty}(\Omega) .
$$

In particular, both $\sigma$ and $|F|^{\frac{p}{p-1}}$ belong to the dual space $\left(W_{0}^{1, p}(\Omega)\right)^{*}$.

(ii) Conversely, suppose that $\sigma=\operatorname{div} F+f$ where $F \in L^{\frac{p}{p-1}}\left(\Omega, \mathbb{R}^{n}\right)$ and $f$ is a locally finite signed measure in $\Omega$ with $|f| \in\left(W_{0}^{1, p}(\Omega)\right)^{*}$ such that

$$
p \int_{\Omega}|F||\varphi|^{p-1}|\nabla \varphi| d x+\int_{\Omega}|\varphi|^{p} d|f| \leq \lambda \int_{\Omega}|\nabla \varphi|^{p} d x \quad \forall \varphi \in C_{c}^{\infty}(\Omega)
$$


for some $\lambda \in\left(0,(p-1)^{p-1}\right)$. Then equation (1.1) has a (possibly sign changing) solution $u \in W_{0}^{1, p}(\Omega)$ such that $e^{\frac{\delta u}{p-1}}-1 \in W_{0}^{1, p}(\Omega)$ for all $\delta \in\left[0, \delta_{0}\right)$ where $\delta_{0}=(p-1) \lambda^{\frac{-1}{p-1}}$. This solution satisfies the Poincaré-Sobolev inequality (1.5) for some $A=A(p)>0$. Moreover, if $\lambda \in\left(0,(p-1)^{\min \{1, p-1\}}\right)$, then both $e^{\frac{u}{p-1}}-1$ and $e^{u}-1$ belong to $W_{0}^{1, p}(\Omega)$.

Several remarks regarding Theorem 1.2 are now in order.

Remark 1.3. By approximation and Fatou's lemma, inequalities (1.6) and (1.7) actually hold for all $\varphi \in W_{0}^{1, p}(\Omega)$. The integral $\int_{\Omega}|\varphi|^{p} d|f|$ makes sense even for $\varphi \in W_{0}^{1, p}(\Omega)$ since $|f|$ is continuous with respect to the capacity $\operatorname{cap}_{p}(\cdot, \Omega)$ and $\varphi$ has a cap -quasicontinuous representative, whose values are defined $\operatorname{cap}_{p}$-quasieverywhere in $\Omega$. Here $\operatorname{cap}_{p}(\cdot, \Omega)$ is the variational p-capacity associated to $\Omega$ defined for each compact set $K \subset \Omega$ by

$$
\operatorname{cap}_{p}(K, \Omega):=\inf \left\{\int_{\Omega}|\nabla \phi|^{p} d x: \phi \in C_{c}^{\infty}(\Omega) \text { and } \phi \geq \chi_{K}\right\} .
$$

Remark 1.4. By Hölder's inequality we see that if $F$ satisfies (1.6) for some $A>0$ then

$$
p \int_{\Omega}|F||\varphi|^{p-1}|\nabla \varphi| d x \leq p A^{\frac{p-1}{p}} \int_{\Omega}|\nabla \varphi|^{p} d x \quad \forall \varphi \in C_{c}^{\infty}(\Omega) .
$$

Thus by Theorem 1.2(ii) if $F \in L^{\frac{p}{p-1}}\left(\Omega, \mathbb{R}^{n}\right)$ satisfies (1.6) for some $0<A<(p-1)^{p} p^{-\frac{p}{p-1}}$ then the equation $-\Delta_{p} u=|\nabla u|^{p}+\operatorname{div} F$ has a solution in $W_{0}^{1, p}(\Omega)$.

Remark 1.5. Let $\mu$ be a nonnegative locally finite measure in $\Omega$. It is well-known that the inequality

$$
\int_{\Omega}|\varphi|^{p} d \mu \leq A_{1} \int_{\Omega}|\nabla \varphi|^{p} d x \quad \forall \varphi \in C_{c}^{\infty}(\Omega)
$$

is equivalent to the condition

$$
\mu(K) \leq A_{2} \operatorname{cap}_{p}(K, \Omega)
$$

for all compact sets $K \subset \Omega$ (see [28, Chapter 2]).

Thus in (ii) of Theorem 1.2, condition (1.7) can be replaced by (1.8) with $\mu=|F|^{\frac{p}{p-1}}+|f|$ for a sufficiently small constant $A_{2}>0$.

Moreover, by (ii) of Theorem 1.2, if $f$ is a locally finite signed measure in $\Omega$ with $|f| \in$ $\left(W_{0}^{1, p}(\Omega)\right)^{*}$ such that $(1.8)$ holds with $d \mu=d|f|$, the we have a decomposition

$$
f=\operatorname{div} F-g
$$

where $F \in L^{\frac{p}{p-1}}\left(\Omega, \mathbb{R}^{n}\right)$ and $g \in L^{1}(\Omega), g \geq 0$, such that the $L^{1}$ function $\mu:=\left(|F|^{\frac{p}{p-1}}+g\right)$ also satisfies (1.8). See [5, 18] for a similar decomposition of measures that are continuous w.r.t the p-capacity. 
Remark 1.6. Let $L^{s, \infty}(\Omega), s \geq 1$, denote the weak $L^{s}$ space on $\Omega$ with quasinorm

$$
\|g\|_{L^{s, \infty}(\Omega)}:=\sup _{t>0} t|\{x \in \Omega:|g(x)|>t\}|^{1 / s} .
$$

For $g \in L^{\frac{n}{p}, \infty}(\Omega)$ with $1<p<n$, it is known that (see, e.g., [16, Eqn. (2.6)])

$$
\int_{\Omega}|\varphi|^{p} g d x \leq S_{n, p}\|g\|_{L^{\frac{n}{p}, \infty}(\Omega)} \int_{\Omega}|\nabla \varphi|^{p} d x \quad \forall \varphi \in C_{c}^{\infty}(\Omega),
$$

where the constant $S_{n, p}$ is given by

$$
S_{n, p}=\left[\frac{p}{\sqrt{\pi}(n-p)}\right]^{p} \Gamma(1+n / 2)^{p / n} .
$$

This shows that in Theorem 1.2(ii), condition (1.7) can be replaced by $|F|^{\frac{p}{p-1}}+|f| \in$ $L^{\frac{n}{p}, \infty}(\Omega)$ with a sufficiently small norm. Existence results under this weak norm condition have been obtained in [16]. See also the earlier works [14,15] where the strong norm condition involving $L^{\frac{n}{p}}(\Omega)$ was used instead. More general existence results in which $|F|^{\frac{p}{p-1}}+|f|$ is assumed to be small in the norm of certain Morrey spaces can be found in the recent paper [29]. Those Morrey space conditions are also stronger than condition (1.7) as they fall into the realm of Fefferman-Phong type conditions (see, e.g., [11, 12, 13, 31, 32]).

We now discuss the Schrödinger type equation with distributional potential (1.3). This equation is interesting in its own right and has a strong connection to equation (1.1) as being observed and exploited, e.g., in [1, 19, 21, 22].

By a solution to (1.3), we mean the following definition.

Definition 1.7. Let $\sigma \in\left(W_{0}^{1, p}(\Omega)\right)^{*}$. A function $v$ defined in $\Omega$ is a solution of (1.3) if $v \geq 0, v-1 \in W_{0}^{1, p}(\Omega), v^{p-1} \in W_{\mathrm{loc}}^{1, p}(\Omega)$, and

$$
\int_{\Omega}|\nabla v|^{p-2} \nabla v \cdot \nabla \varphi d x=(p-1)^{1-p}\left\langle\sigma, v^{p-1} \varphi\right\rangle \quad \forall \varphi \in C_{c}^{\infty}(\Omega) .
$$

Note that the right hand side of (1.9) makes sense since $v^{p-1} \varphi \in W_{0}^{1, p}(\Omega)$ and $\sigma \in$ $\left(W_{0}^{1, p}(\Omega)\right)^{*}$.

Formally, by making the change of unknowns $v=e^{\frac{u}{p-1}}$, equation (1.1) is transformed into the Schrödinger type equation (1.3). Indeed, it is possible to show rigorously that Theorem 1.2 implies the existence of finite energy solutions to (1.3): 
Theorem 1.8. Suppose that $\sigma=\operatorname{div} F+f$ where $F \in L^{\frac{p}{p-1}}\left(\Omega, \mathbb{R}^{n}\right)$ and $f$ is a locally finite signed measure in $\Omega$ with $|f| \in\left(W_{0}^{1, p}(\Omega)\right)^{*}$ such that

$$
p \int_{\Omega}|F||\varphi|^{p-1}|\nabla \varphi| d x+\int_{\Omega}|\varphi|^{p} d|f| \leq \lambda \int_{\Omega}|\nabla \varphi|^{p} d x \quad \forall \varphi \in C_{c}^{\infty}(\Omega),
$$

for some $\lambda \in\left(0,(p-1)^{\min \{1, p-1\}}\right)$. Then equation (1.3) has a nonnegative solution $v$ such that both $v-1$ and $v^{p-1}-1$ belong to $W_{0}^{1, p}(\Omega)$. Moreover, $v$ satisfies the following PoincaréSobolev inequality

$$
\int_{\Omega}\left|\frac{\nabla v}{v}\right|^{p}|\varphi|^{p} d x \leq A \int_{\Omega}|\nabla \varphi|^{p} d x \quad \forall \varphi \in C_{c}^{\infty}(\Omega)
$$

with a constant $A=A(p)>0$.

Remark 1.9. If the factor $(p-1)^{1-p}$ on the right-hand side of (1.3) is dropped then the smallness condition on $\lambda$ becomes $\lambda \in\left(0, p^{\#}\right)$, where $p^{\#}=(p-1)^{2-p}$ if $p>2$ and $p^{\#}=1$ if $p \leq 2$ as in [22]. The sharpness of $p^{\#}$ (and that of $(p-1)^{\min \{1, p-1\}}$ for (1.3)) was also justified in [22].

One could also treat the Schrödinger type equation (1.3) in a more general fashion, where the standard $p$-Laplacian is replaced by a quasilinear elliptic operator with merely measurable 'coefficients'. See Remark 6.1 below and see also [22].

We mention that the existence of finite energy solutions to (1.3) in the case $\sigma \geq 0$ was obtained in [19] by a method that does not seem to work for sign changing $\sigma$ (see also [1] for $p=2$ ). On the other hand, the work [22] (see also [21]) obtains a locally finite energy solution $v \in W_{\text {loc }}^{1, p}(\Omega)$ to the first two equations in (1.3) (without any boundary conditions) only under the mild restriction

$$
-\Lambda \int_{\Omega}|\nabla \varphi|^{p} d x \leq\left\langle\sigma,|\varphi|^{p}\right\rangle \leq \lambda \int_{\Omega}|\nabla \varphi|^{p} d x \quad \text { for all } \varphi \in C_{c}^{\infty}(\Omega)
$$

for some $\lambda \in\left(0,(p-1)^{\min \{1, p-1\}}\right)$ and $\Lambda \in(0,+\infty)$. Moreover, $v$ also satisfies (1.10) for some $A>0$. Then, also under the restriction $\lambda \in\left(0,(p-1)^{\min \{1, p-1\}}\right)$, by the logarithmic transformation $u=(p-1) \log (v)$ it was obtained in [22], a solution $u \in W_{\text {loc }}^{1, p}(\Omega)$ to the first equation in (1.1) (but without any boundary condition) that also satisfies (1.5) for some $A>0$.

In this paper, we follow an opposite route, i.e., we first treat equation (1.1) directly and then deduce existence for the Schrödinger type equation (1.3) from it. This way, we are able to treat equation (1.1) in its most general form, i.e., the nonlinear equation with general 
structure (1.2). Moreover, for equation (1.1) we obtain larger upper bound for $\lambda$ in the existence condition (1.7) (i.e., $(p-1)^{p-1}$ versus $\left.(p-1)^{\min \{1, p-1\}}\right)$. Our approach to (1.2) is a refinement of the approach of V. Ferone and F. Murat in $[15,16]$. The main difficulties to overcome here are the generality nature of $\sigma$ and the sharpness of the smallness constants. In particular, in this scenario one does not gain any higher integrability on the nonlinear term $\mathcal{B}(x, u, \nabla u)$, which makes it impossible to follow a compactness argument as in [29]. Moreover, in order for us to apply the existence results of (1.1) to (1.3) we need to find a solution $u$ of (1.1) with the additional property that both $e^{\frac{u}{p-1}}-1$ and $e^{u}-1$ belong to $W_{0}^{1, p}(\Omega)$ as stated in Theorem 1.2 .

\section{Equations With General NONLINEAR StRUCture}

As we have mentioned, existence results in the spirit of Theorem 1.2(ii) also hold for equations with a more general nonlinear structure (1.2). For that we need the following assumptions on the nonlinearities $\mathcal{A}$ and $\mathcal{B}$ :

Assumption on $\mathcal{A}$. The nonlinearity $\mathcal{A}: \Omega \times \mathbb{R} \times \mathbb{R}^{n} \rightarrow \mathbb{R}^{n}$ is a Carathédory function, i.e., $\mathcal{A}(x, s, \xi)$ is measurable in $x$ for every $(s, \xi)$ and continuous in $(s, \xi)$ for a.e. $x \in \Omega$. For some $p>1$, it holds that

$$
\begin{gathered}
\langle\mathcal{A}(x, s, \xi)-\mathcal{A}(x, s, \eta), \xi-\eta\rangle>0 \\
\langle\mathcal{A}(x, s, \xi), \xi\rangle \geq \alpha_{0}|\xi|^{p} \\
|\mathcal{A}(x, s, \xi)| \leq a_{0}|\xi|^{p-1}+a_{1}|s|^{p-1}
\end{gathered}
$$

for every $(\xi, \eta) \in \mathbb{R}^{n} \times \mathbb{R}^{n}, \xi \neq \eta$, and a.e. $x \in \Omega$. Here $\alpha_{0}>0$, and $a_{0}, a_{1} \geq 0$.

Assumption on $\mathcal{B}$. The nonlinearity $\mathcal{B}: \Omega \times \mathbb{R} \times \mathbb{R}^{n} \rightarrow \mathbb{R}$ is a Carathédory function which satisfies, for a.e. $x \in \Omega$, every $s \in \mathbb{R}$, and every $\xi \in \mathbb{R}^{n}$,

$$
|\mathcal{B}(x, s, \xi)| \leq b_{0}|\xi|^{p}+b_{1}|s|^{m}, \quad \mathcal{B}(x, s, \xi) \operatorname{sign}(s) \leq \alpha_{0} \gamma_{0}|\xi|^{p},
$$

where $m>0$, and $b_{0}, b_{1}, \gamma_{0} \geq 0$. Here $\alpha_{0}$ is as given in (2.2).

By a solution of (1.2) we mean the following.

Definition 2.1. Under (2.1)-(2.4), a function $u \in W_{0}^{1, p}(\Omega)$ is a solution of (1.2) if $\mathcal{B}(x, u, \nabla u) \in L_{\mathrm{loc}}^{1}(\Omega)$ and

$$
\int_{\Omega} \mathcal{A}(x, u, \nabla u) \cdot \nabla \varphi d x=\int_{\Omega} \mathcal{B}(x, u, \nabla u) \varphi d x+\langle\sigma, \varphi\rangle
$$

holds for all test functions $\varphi \in C_{c}^{\infty}(\Omega)$. 
We remark that in the case $\mathcal{B}(x, u, \nabla u) \in L^{1}(\Omega)$ and $\sigma \in\left(W_{0}^{1, p}(\Omega)\right)^{*}$, we can take any function $\varphi \in W_{0}^{1, p}(\Omega) \cap L^{\infty}(\Omega)$ as a test function in the above definition. This follows from a result of Brézis and Browder [9] as we have $\mathcal{B}(x, u, \nabla u) \in\left(W_{0}^{1, p}(\Omega)\right)^{*} \cap L^{1}(\Omega)$. It can also be seen by approximating $\varphi$ in $W_{0}^{1, p}(\Omega)$ by a sequence $\varphi_{j} \in C_{c}^{\infty}(\Omega)$ such that $\left|\varphi_{j}\right| \leq|\varphi| \leq M$ a.e. (using Theorem 9.3.1 in [2] and suitable convolutions).

We mention that in the special case $|\mathcal{B}(x, u, \nabla u)| \in\left(W_{0}^{1, p}(\Omega)\right)^{*} \cap L^{1}(\Omega)$, we can even drop the condition $\varphi \in L^{\infty}(\Omega)$. In fact, we have the following more general result.

Lemma 2.2. Suppose that $f$ is a locally finite signed measure in $\Omega$ with $|f| \in\left(W_{0}^{1, p}(\Omega)\right)^{*}$. Then for any $\varphi \in W_{0}^{1, p}(\Omega)$ we have

$$
\langle f, \varphi\rangle=\int_{\Omega} \widetilde{\varphi} d f
$$

where $\widetilde{\varphi}$ is any cap $_{p}$-quasicontinuous representative of $\varphi$.

In the case $f$ is nonnegative, the proof of Lemma 2.2 can be found in [30, Lemma 2.5]. The general case also follows from that, since $f=f^{+}+f^{-}$and both $f^{+}$and $f^{-}$belong to $\left(W_{0}^{1, p}(\Omega)\right)^{*}$. In what follows, when dealing with pointwise behavior of functions in $W_{0}^{1, p}(\Omega)$ we will implicitly use their $\operatorname{cap}_{p}$-quasicontinuous representatives. Lemma 2.2 will be used, e.g., in (3.11) below.

Under the above assumptions on $\mathcal{A}$ and $\mathcal{B}$, we obtain the following existence result.

Theorem 2.3. Let $\sigma=\operatorname{div} F+f$ where $F \in L^{\frac{p}{p-1}}\left(\Omega, \mathbb{R}^{n}\right)$ and $f$ is a locally finite signed measure in $\Omega$ with $|f| \in\left(W_{0}^{1, p}(\Omega)\right)^{*}$ such that

$$
p \int_{\Omega}|F||\varphi|^{p-1}|\nabla \varphi| d x+\int_{\Omega}|\varphi|^{p} d|f| \leq \lambda \int_{\Omega}|\nabla \varphi|^{p} d x
$$

holds for all $\varphi \in C_{c}^{\infty}(\Omega)$, with $\lambda \in\left(0, \gamma_{0}^{1-p} \alpha_{0}(p-1)^{p-1}\right)$. Then there exists a solution $u \in W_{0}^{1, p}(\Omega)$ to the equation

$$
-\operatorname{div} \mathcal{A}(x, u, \nabla u)=\mathcal{B}(x, u, \nabla u)+\sigma \quad \text { in } \quad \Omega,
$$

such that $e^{\frac{\delta|u|}{p-1}}-1 \in W_{0}^{1, p}(\Omega)$ for all $\delta \in\left[\gamma_{0}, \delta_{0}\right)$, with $\delta_{0}=(p-1)\left(\frac{\alpha_{0}}{\lambda}\right)^{\frac{1}{p-1}}$.

Moreover, for any $\delta_{1}>\gamma_{0}$ such that (2.5) holds with

$$
\lambda<\left(\frac{p-1}{\delta_{1}}\right)^{p} \alpha_{0}\left(\frac{\delta_{1}}{p-1}+\delta_{1}-\gamma_{0}\right)
$$

we have $e^{\frac{\delta_{1}|u|}{p-1}}-1 \in W_{0}^{1, p}(\Omega)$. 
Remark 2.4. It is easy to check that, for $\delta_{1}>\gamma_{0}$ one has

$$
\left(\frac{p-1}{\delta_{1}}\right)^{p} \alpha_{0}\left(\frac{\delta_{1}}{p-1}+\delta_{1}-\gamma_{0}\right)<\gamma_{0}^{1-p} \alpha_{0}(p-1)^{p-1}
$$

Moreover, for example with $p>2$ and $\alpha_{0}=\gamma_{0}=1$, if (2.5) holds with $\lambda<p-1 \in$ $\left(0,(p-1)^{p-1}\right)$, then we see that $(3.2)$ holds with $1 \leq \delta<(p-1)(p-1)^{\frac{-1}{p-1}}$, but it does not allow us to take $\delta=p-1$ ! On the other hand, for $\lambda<p-1$ inequality (2.7) holds with $\delta_{1}=p-1$ and thus $e^{|u|}-1 \in W_{0}^{1, p}(\Omega)$.

Due to the general structures of $\mathcal{A}$ and $\mathcal{B}$, here we do not claim that the solution $u$ obtained in Theorem 2.3 satisfies the Poincaré-Sobolev inequality (1.5).

The paper is organized as follows: In Section 5, we provide the proof of Theorem 2.3. This proof is based on the existence of solutions to an approximate equation along with certain uniform bounds given in Section 4. These important uniform bounds are in turn deduced from the a priori estimate of Section 3, though not directly. Finally, the proof of Theorems 1.2 and 1.8 will be given in Section 6 .

\section{An A PRIORI EStimate}

In this section, we obtain certain exponential type a priori bounds for solutions of

$$
-\operatorname{div} \mathcal{A}(x, u, \nabla u)+\varepsilon|u|^{p-2} u=\mathcal{B}(x, u, \nabla u)+\sigma \quad \text { in } \quad \Omega,
$$

where $\varepsilon \geq 0$. The case $\varepsilon>0$ will be needed in the next section to absorb certain unfavorable terms in the approximating process; see (4.9) below. Earlier, this idea was implemented by V. Ferone and F. Murat in [16].

Theorem 3.1. Let $\sigma=\operatorname{div} F+f$ where $F \in L^{\frac{p}{p-1}}\left(\Omega, \mathbb{R}^{n}\right)$ and $f$ is a locally finite signed measure in $\Omega$ with $|f| \in\left(W_{0}^{1, p}(\Omega)\right)^{*}$ such that (2.5) holds for all $\varphi \in C_{c}^{\infty}(\Omega)$, with $\lambda \in$ $\left(0, \gamma_{0}^{1-p} \alpha_{0}(p-1)^{p-1}\right)$. Then for any $\varepsilon \geq 0$ and any $W_{0}^{1, p}(\Omega)$ solution $u$ to equation (3.1) such that $e^{\frac{\delta|u|}{p-1}}-1 \in W_{0}^{1, p}(\Omega)$, we have

$$
\|u\|_{W_{0}^{1, p}(\Omega)}+\left\|e^{\frac{\delta|u|}{p-1}}-1\right\|_{W_{0}^{1, p}(\Omega)} \leq M_{\delta}
$$

provided $\delta \in\left[\gamma_{0}, \delta_{0}\right)$ where $\delta_{0}=(p-1)\left(\alpha_{0} / \lambda\right)^{\frac{1}{p-1}}$. Here $M_{\delta}$ is independent of $u$ and $\varepsilon$.

Moreover, for any $\delta_{1}>\gamma_{0}$ such that $e^{\frac{\delta_{1}|u|}{p-1}}-1 \in W_{0}^{1, p}(\Omega)$, and (2.5) holds with $\lambda$ satisfying (2.7), we have

$$
\left\|e^{\frac{\delta_{1}|u|}{p-1}}-1\right\|_{W_{0}^{1, p}(\Omega)} \leq M_{\delta_{1}}+C_{\delta_{1}}\|\nabla u\|_{L^{p}(\Omega)}
$$


The constants $M_{\delta_{1}}$ and $C_{\delta_{1}}$ are independent of $u$ and $\varepsilon$.

Proof. Let $u \in W_{0}^{1, p}(\Omega)$ be a solution of (3.1) and define

$$
w=\operatorname{sign}(u)\left[e^{\mu|u|}-1\right] / \mu, \quad \text { with } \mu=\delta /(p-1),
$$

where $\operatorname{sign}(u)=0$ if $u=0, \operatorname{sign}(u)=1$ if $u>0$, and $\operatorname{sign}(u)=-1$ if $u<0$. Then from the assumption $e^{\mu|u|}-1 \in W_{0}^{1, p}(\Omega)$, we see that $w \in W_{0}^{1, p}(\Omega)$ with

$$
\nabla w=e^{\mu|u|} \nabla u
$$

Indeed, for $\varepsilon>0$ define $f_{\varepsilon}(x)=\frac{x}{\sqrt{x^{2}+\varepsilon^{2}}}, x \in \mathbb{R}$, and denote by $T_{s}, s>0$, the two-sided truncation operator at level $s$, i.e.,

$$
T_{s}(r)=r \text { if }|r| \leq s \quad \text { and } \quad T_{s}(r)=\operatorname{sign}(r) s \text { if }|r|>s,
$$

then it follows that $T_{s}(u) \in W_{0}^{1, p}(\Omega)$ for any $s>0$ and

$$
\begin{aligned}
\nabla\left[f_{\varepsilon}\left(T_{s}(u)\right)\left(e^{\mu\left|T_{s}(u)\right|}-1\right) / \mu\right]= & \frac{\nabla T_{s}(u) \varepsilon^{2}}{\left(T_{s}(u)^{2}+\varepsilon^{2}\right)^{3 / 2}}\left(e^{\mu\left|T_{s}(u)\right|}-1\right) / \mu \\
& +f_{\varepsilon}\left(T_{s}(u)\right) \nabla\left(e^{\mu\left|T_{s}(u)\right|}-1\right) / \mu
\end{aligned}
$$

in the weak sense. Note that

$$
\begin{gathered}
\varepsilon^{2}\left(e^{\mu\left|T_{s}(u)\right|}-1\right) / \mu \leq \frac{e^{\mu s}}{\mu s} \varepsilon^{2}\left|T_{s}(u)\right| \leq \frac{e^{\mu s}}{\mu s}\left(\left|T_{s}(u)\right|^{2}+\varepsilon^{2}\right)^{3 / 2}, \\
f_{\varepsilon}\left(T_{s}(u)\right) \rightarrow \operatorname{sign}\left(T_{s}(u)\right)=\operatorname{sign}(u) \text { as } \varepsilon \rightarrow 0^{+},
\end{gathered}
$$

and thus by Dominated Convergence Theorem we find

$$
\nabla\left[\operatorname{sign}(u)\left(e^{\mu\left|T_{s}(u)\right|}-1\right) / \mu\right]=\operatorname{sign}(u) \nabla\left(e^{\mu\left|T_{s}(u)\right|}-1\right) / \mu=e^{\mu\left|T_{s}(u)\right|} \nabla T_{s}(u) .
$$

Now using the assumption $e^{\mu|u|}|\nabla u| \in L^{p}(\Omega)$ and letting $s \rightarrow \infty$, we obtain (3.4).

For each $s>0$, we will use the following test function for (3.1):

$$
v_{s}=e^{\delta\left|u_{s}\right|} w_{s}
$$

where $u_{s}=T_{s}(u)$ and $w_{s}=\operatorname{sign}(u)\left[e^{\mu\left|u_{s}\right|}-1\right] / \mu$ with $\mu=\delta /(p-1)$.

From the definition of $w_{s}$ we have $\left|w_{s}\right| \leq|w|$ and $\nabla w_{s}=e^{\mu\left|u_{s}\right|} \nabla u_{s}$. Thus both $w_{s}$ and $v_{s}$ belong to $W_{0}^{1, p}(\Omega) \cap L^{\infty}(\Omega)$ and moreover,

$$
\nabla v_{s}=\left[e^{\delta|u|} \nabla w+\delta|w| e^{\delta|u|} \nabla u\right] \chi_{\{|u| \leq s\}}
$$


Using $v_{s}$ as a test function in (3.1), we get

$$
\begin{aligned}
\int_{\Omega} \mathcal{A}(x, u, \nabla u) \cdot \nabla w e^{\delta|u|} \chi_{\{|u| \leq s\}} d x+\varepsilon \int_{\Omega}|u|^{p-2} u e^{\delta\left|u_{s}\right|} w_{s} d x \\
=-\int_{\Omega} \delta|w| e^{\delta|u|} \mathcal{A}(x, u, \nabla u) \cdot \nabla u \chi_{\{|u| \leq s\}} d x+ \\
\quad+\int_{\Omega} \mathcal{B}(x, u, \nabla u) e^{\delta\left|u_{s}\right|} w_{s} d x+\int_{\Omega} F \cdot \nabla v_{s} d x+\int_{\Omega} v_{s} d f .
\end{aligned}
$$

We now write this equality as

$$
I_{1}+I_{2}=I_{3}+I_{4}+I_{5}+I_{6}
$$

where $I_{i}, i \in\{1, \ldots, 6\}$, are the corresponding terms.

Estimate for $I_{1}$ : Since $\nabla w_{s}=e^{\mu\left|u_{s}\right|} \nabla u_{s}$, using the coercivity condition (2.2), we see that

$$
\begin{aligned}
I_{1} & =\int_{\Omega} \mathcal{A}\left(x, u_{s}, \nabla u_{s}\right) \cdot \nabla w_{s} e^{\delta\left|u_{s}\right|} \chi_{\{|u| \leq s\}} d x \\
& =\int_{\Omega} \mathcal{A}\left(x, u_{s}, \nabla u_{s}\right) \cdot \nabla u_{s} e^{(\mu+\delta)\left|u_{s}\right|} d x \\
& \geq \alpha_{0} \int_{\Omega}\left|\nabla w_{s}\right|^{p} d x,
\end{aligned}
$$

where we used the fact $\mu+\delta=p \mu$.

Estimate for $I_{2}$ : We have

$$
I_{2}=\varepsilon \int_{\Omega}|u|^{p-1} e^{\delta\left|u_{s}\right|} \frac{e^{\mu\left|u_{s}\right|}-1}{\mu} d x \geq \varepsilon s^{p-1} \int_{\Omega} e^{\delta s} \frac{e^{\mu s}-1}{\mu} \chi_{\{|u|>s\}} \geq 0 .
$$

Estimate for $I_{3}+I_{4}$ : By $(2.2)$ we have

$$
\begin{aligned}
I_{3}+I_{4}= & -\int_{\Omega} \delta|w| e^{\delta|u|} \mathcal{A}(x, u, \nabla u) \cdot \nabla u \chi_{\{|u| \leq s\}} d x+\int_{\Omega} \mathcal{B}(x, u, \nabla u) e^{\delta\left|u_{s}\right|} w_{s} d x \\
\leq & -\int_{\Omega} \delta \alpha_{0}\left|w_{s}\right| e^{\delta\left|u_{s}\right|}\left|\nabla u_{s}\right|^{p} d x+\int_{\Omega} \mathcal{B}(x, u, \nabla u) \operatorname{sign}(u) e^{\delta\left|u_{s}\right|}\left|w_{s}\right| d x \\
= & -\int_{\Omega} \delta \alpha_{0}\left|w_{s}\right| e^{\delta\left|u_{s}\right|}\left|\nabla u_{s}\right|^{p} d x+ \\
& +\int_{\Omega} \mathcal{B}(x, u, \nabla u) \operatorname{sign}(u) e^{\delta\left|u_{s}\right|}\left|w_{s}\right|\left[\chi_{\{|u| \leq s\}}+\chi_{\{|u|>s\}}\right] d x
\end{aligned}
$$

Since we assume $\delta \geq \gamma_{0}$, this and the second condition in (2.4) imply that

$$
\begin{aligned}
I_{3}+I_{4} \leq & \int_{\Omega}\left(-\delta+\gamma_{0}\right) \alpha_{0}\left|w_{s}\right| e^{\delta\left|u_{s}\right|}\left|\nabla u_{s}\right|^{p} d x \\
& +\int_{\Omega} \mathcal{B}(x, u, \nabla u) \operatorname{sign}(u) e^{\delta\left|u_{s}\right|}\left|w_{s}\right| \chi_{\{|u|>s\}} d x \\
\leq & \int_{\Omega} \mathcal{B}(x, u, \nabla u) \operatorname{sign}(u) e^{\delta\left|u_{s}\right|}\left|w_{s}\right| \chi_{\{|u|>s\}} d x
\end{aligned}
$$


Thus by the first inequality on (2.4) and the fact that

$$
\begin{aligned}
\left|v_{s}\right| & =e^{\delta\left|u_{s}\right|}\left|w_{s}\right|=\left(1+\mu\left|w_{s}\right|\right)^{\delta / \mu}\left|w_{s}\right|=\left(1+\mu\left|w_{s}\right|\right)^{p-1}\left|w_{s}\right| \\
& \leq \frac{1}{\mu}\left(1+\mu\left|w_{s}\right|\right)^{p} \leq \frac{1}{\mu} e^{p \mu\left|u_{s}\right|} \leq \frac{1}{\mu} e^{p \mu|u|},
\end{aligned}
$$

we find

$$
\begin{aligned}
I_{3}+I_{4} & \leq \int_{\Omega}\left(b_{0}|\nabla u|^{p}+b_{1}|u|^{m}\right) \frac{1}{\mu} e^{p \mu|u|} \chi_{\{|u|>s\}} d x \\
& =\frac{1}{\mu} \int_{\Omega} b_{0}|\nabla w|^{p} \chi_{\{|u|>s\}} d x+\frac{1}{\mu} \int_{\Omega} b_{1}|u|^{m} e^{p \mu|u|} \chi_{\{|u|>s\}} d x .
\end{aligned}
$$

Estimate for $I_{5}+I_{6}$ : Using (3.9) again and Lemma 2.2 we have

$$
\begin{aligned}
I_{5}+I_{6}= & \int_{\Omega} F \cdot \nabla\left[\left(1+\mu\left|w_{s}\right|\right)^{p-1} w_{s}\right] d x+\int_{\Omega} v_{s} d f \\
= & \int_{\Omega} F \cdot\left[(p-1)\left(1+\mu\left|w_{s}\right|\right)^{p-2} \nabla w_{s} \operatorname{sign}\left(w_{s}\right) \mu w_{s}\right] d x+ \\
& +\int_{\Omega} F \cdot\left[\left(1+\mu\left|w_{s}\right|\right)^{p-1} \nabla w_{s}\right] d x+\int_{\Omega} v_{s} d f \\
\leq & p \int_{\Omega}|F|\left(1+\mu\left|w_{s}\right|\right)^{p-1}\left|\nabla w_{s}\right| d x+\int_{\Omega}\left(1+\mu\left|w_{s}\right|\right)^{p-1}\left|w_{s}\right| d|f| .
\end{aligned}
$$

Using the inequality

$$
\left(1+\mu\left|w_{s}\right|\right)^{p-1} \leq(1+\tilde{\varepsilon}) \mu^{p-1}\left|w_{s}\right|^{p-1}+C(\tilde{\varepsilon}, p), \quad \tilde{\varepsilon}>0,
$$

and Hölder's inequality we have

$$
\begin{aligned}
I_{5}+I_{6} \leq & (1+\tilde{\varepsilon}) \mu^{p-1} p \int_{\Omega}|F|\left|w_{s}\right|^{p-1}\left|\nabla w_{s}\right| d x+(1+\tilde{\varepsilon}) \mu^{p-1} \int_{\Omega}\left|w_{s}\right|^{p} d|f| \\
& +C(\tilde{\varepsilon}, p)\left(\|F\|_{L^{\frac{p}{p-1}(\Omega)}}+\||f|\|_{\left(W_{0}^{1, p}(\Omega)\right)^{*}}\right)\left\|\nabla w_{s}\right\|_{L^{p}(\Omega)} .
\end{aligned}
$$

We recall that by approximation and Fatou's lemma (2.5) holds for all $\varphi \in W_{0}^{1, p}(\Omega)$. Then by (2.5) we get

$$
\begin{aligned}
I_{5}+I_{6} \leq & (1+\tilde{\varepsilon}) \mu^{p-1} \lambda\left\|\nabla w_{s}\right\|_{L^{p}(\Omega)}^{p}+ \\
& +C(\tilde{\varepsilon}, p)\left(\|F\|_{L^{\frac{p}{p-1}(\Omega)}}+\|f \mid\|_{\left(W_{0}^{1, p}(\Omega)\right)^{*}}\right)\left\|\nabla w_{s}\right\|_{L^{p}(\Omega)} .
\end{aligned}
$$


We now use estimates (3.6), (3.7), (3.10) and (3.12) in equality (3.5) to obtain the following bound

$$
\begin{aligned}
\kappa(\varepsilon)\left\|\nabla w_{s}\right\|_{L^{p}(\Omega)}^{p} \leq & \frac{1}{\mu} \int_{\Omega} b_{0}|\nabla w|^{p} \chi_{\{|u|>s\}} d x+\frac{1}{\mu} \int_{\Omega} b_{1}|u|^{m} e^{p \mu|u|} \chi_{\{|u|>s\}} d x+ \\
& +C(\tilde{\varepsilon}, p)\left(\|F\|_{L^{\frac{p}{p-1}(\Omega)}}+\||f|\|_{\left(W_{0}^{1, p}(\Omega)\right)^{*}}\right)\left\|\nabla w_{s}\right\|_{L^{p}(\Omega)},
\end{aligned}
$$

where $\kappa(\varepsilon)=\alpha_{0}-(1+\varepsilon) \mu^{p-1} \lambda$. Observe that when $\delta<\delta_{0}=(p-1)\left(\alpha_{0} / \lambda\right)^{\frac{1}{p-1}}$ we have

$$
\mu^{p-1} \lambda=(\delta /(p-1))^{p-1} \lambda<\left(\delta_{0} /(p-1)\right)^{p-1} \lambda=\alpha_{0}
$$

and thus we can choose $\varepsilon>0$ small enough so that $\kappa(\varepsilon)>0$.

Since $\left(e^{\mu|u|}-1\right) \in W_{0}^{1, p}(\Omega)$, by Sobolev's embedding theorem it holds that $e^{p \mu|u|} \in$ $L^{\frac{n}{n-p}}(\Omega)$ if $1<p<n$ and $e^{p \mu|u|} \in L^{2}(\Omega)$, say, if $p \geq n$. Thus we have $|u|^{m} e^{p \mu|u|} \in L^{1}(\Omega)$. Now letting $s \nearrow \infty$ in the last inequality, we find

$$
\|\nabla w\|_{L^{p}(\Omega)}^{p} \leq C\left(\|F\|_{L^{\frac{p}{p-1}(\Omega)}}+\||f|\|_{\left(W_{0}^{1, p}(\Omega)\right)^{*}}\right)\|\nabla w\|_{L^{p}(\Omega)}
$$

which yields

$$
\left\|e^{\delta|u| /(p-1)}-1\right\|_{W_{0}^{1, p}(\Omega)} \leq C(\delta, \lambda, p)\left(\|F\|_{L^{\frac{p}{p-1}(\Omega)}}+\||f|\|_{\left(W_{0}^{1, p}(\Omega)\right)^{*}}\right)^{\frac{1}{p-1}} .
$$

Finally, note that

$$
\|u\|_{W_{0}^{1, p}(\Omega)}=\|\nabla u\|_{L^{p}(\Omega)} \leq \frac{p-1}{\delta}\left\|\nabla\left(e^{\delta|u| /(p-1)}-1\right)\right\|_{L^{p}(\Omega)}
$$

and hence, we also have

$$
\|u\|_{W_{0}^{1, p}(\Omega)} \leq C(\delta, \lambda, p)\left(\|F\|_{L^{\frac{p}{p-1}(\Omega)}}+\||f|\|_{\left(W_{0}^{1, p}(\Omega)\right)^{*}}\right)^{\frac{1}{p-1}} .
$$

This proves inequality (3.2) for all $\delta \in\left[\gamma_{0}, \delta_{0}\right)$.

To prove inequality (3.3) for $\delta_{1}$, we first define $\mu_{1}=\frac{\delta_{1}}{p-1}$ and redefine

$$
w=\operatorname{sign}(u)\left[e^{\mu_{1}|u|}-1\right] / \mu_{1}, \quad w_{s}=\operatorname{sign}(u)\left[e^{\mu_{1}\left|u_{s}\right|}-1\right] / \mu_{1} .
$$

Observe that

$$
\left(e^{\mu_{1}\left|u_{s}\right|}-1\right) e^{\delta_{1}\left|u_{s}\right|} \geq(1-\varepsilon) e^{\left(\delta_{1}+\mu_{1}\right)\left|u_{s}\right|}-C\left(\varepsilon, \delta_{1}\right) \quad \text { for all } \varepsilon \in(0,1),
$$


and thus by the first inequality in $(3.8)$, with $\left(\delta_{1}, \mu_{1}\right)$ in place of $(\delta, \mu)$, we have

$$
\begin{aligned}
I_{3}+I_{4} \leq & \int_{\Omega}\left(-\delta_{1}+\gamma_{0}\right) \frac{\alpha_{0}}{\mu_{1}}\left(e^{\mu_{1}\left|u_{s}\right|}-1\right) e^{\delta_{1}\left|u_{s}\right|}\left|\nabla u_{s}\right|^{p} d x \\
& +\int_{\Omega} \mathcal{B}(x, u, \nabla u) \operatorname{sign}(u) e^{\delta_{1}\left|u_{s}\right|}\left|w_{s}\right| \chi_{\{|u|>s\}} d x \\
\leq & \int_{\Omega}(1-\varepsilon)\left(-\delta_{1}+\gamma_{0}\right) \frac{\alpha_{0}}{\mu_{1}}\left|\nabla w_{s}\right|^{p} d x+\int_{\Omega} C\left(\varepsilon, \delta_{1}\right)\left(\delta_{1}-\gamma_{0}\right) \frac{\alpha_{0}}{\mu_{1}}\left|\nabla u_{s}\right|^{p} d x \\
& +\int_{\Omega} \mathcal{B}(x, u, \nabla u) \operatorname{sign}(u) e^{\delta_{1}\left|u_{s}\right|}\left|w_{s}\right| \chi_{\{|u|>s\}} d x .
\end{aligned}
$$

Here in the last inequality we used that $\delta_{1}>\gamma_{0}$ and $\left|\nabla w_{s}\right|^{p}=e^{\left(\delta_{1}+\mu_{1}\right)\left|u_{s}\right|}\left|\nabla u_{s}\right|^{p}$.

Thus arguing as in (3.10) for the last term we find

$$
\begin{aligned}
I_{3}+I_{4} \leq & \int_{\Omega}(1-\varepsilon)\left(-\delta_{1}+\gamma_{0}\right) \frac{\alpha_{0}}{\mu_{1}}\left|\nabla w_{s}\right|^{p} d x+C(\varepsilon) \int_{\Omega}\left|\nabla u_{s}\right|^{p} d x \\
& +\frac{1}{\mu_{1}} \int_{\Omega} b_{0}|\nabla w|^{p} \chi_{\{|u|>s\}} d x+\frac{1}{\mu_{1}} \int_{\Omega} b_{1}|u|^{m} e^{p \mu_{1}|u|} \chi_{\{|u|>s\}} d x .
\end{aligned}
$$

Using estimates $(3.6),(3.7),(3.12)$ (with $\left(\delta_{1}, \mu_{1}\right)$ in place of $\left.(\delta, \mu)\right)$ and $(3.14)$ in equality (3.5) we then get

$$
\begin{aligned}
\kappa_{1}(\varepsilon)\left\|\nabla w_{s}\right\|_{L^{p}(\Omega)}^{p} \leq & \frac{1}{\mu_{1}} \int_{\Omega} b_{0}|\nabla w|^{p} \chi_{\{|u|>s\}} d x+\frac{1}{\mu_{1}} \int_{\Omega} b_{1}|u|^{m} e^{p \mu_{1}|u|} \chi_{\{|u|>s\}} d x+ \\
& +C(\varepsilon)\left(\|F\|_{L^{\frac{p}{p-1}(\Omega)}}+\||f|\|_{\left(W_{0}^{1, p}(\Omega)\right)^{*}}\right)\left\|\nabla w_{s}\right\|_{L^{p}(\Omega)}+ \\
& +C(\varepsilon) \int_{\Omega}\left|\nabla u_{s}\right|^{p} d x
\end{aligned}
$$

where $\kappa_{1}(\varepsilon)=\alpha_{0}+(1-\varepsilon)\left(\delta_{1}-\gamma_{0}\right) \frac{\alpha_{0}}{\mu_{1}}-(1+\varepsilon) \mu_{1}^{p-1} \lambda$, with $\varepsilon \in(0,1)$. Thus when $(2.7)$ holds we can find $\varepsilon \in(0,1)$ such that $\kappa_{1}(\varepsilon)>0$. Then using Young's inequality and letting $s \rightarrow \infty$ we eventually obtain

$$
\|\nabla w\|_{L^{p}(\Omega)}^{p} \leq C\left(\|F\|_{L^{\frac{p}{p-1}(\Omega)}}+\||f|\|_{\left(W_{0}^{1, p}(\Omega)\right)^{*}}\right)^{\frac{1}{p-1}}+C \int_{\Omega}|\nabla u|^{p} d x .
$$

This proves inequality (3.3) for all $\delta_{1}>\gamma_{0}$ such (2.7) holds.

\section{Existence of solutions to an approximate EQUATion}

For $k>0$, we now define a function $\mathcal{H}_{k}(x, s, \xi)$ by letting

$$
\mathcal{H}_{k}(x, s, \xi):=\frac{\mathcal{B}(x, s, \xi)}{1+\frac{1}{k}|\mathcal{B}(x, s, \xi)|} \text {. }
$$

Note $\left|\mathcal{H}_{k}(x, s, \xi)\right| \leq k$, and (2.4) also holds with $\mathcal{H}_{k}(x, s, \xi)$ in place of $\mathcal{B}(x, s, \xi)$. Moreover,

$$
\lim _{k \rightarrow \infty} \mathcal{H}_{k}(x, s, \xi)=\mathcal{B}(x, s, \xi)
$$


The goal of this section is to obtain existence results for the approximate equation

$$
-\operatorname{div} \mathcal{A}(x, u, \nabla u)=\mathcal{H}_{k}(x, u, \nabla u)+\sigma \quad \text { in } \quad \Omega
$$

Proposition 4.1. Let $\sigma=\operatorname{div} F+f$ where $F \in L^{\frac{p}{p-1}}\left(\Omega, \mathbb{R}^{n}\right)$ and $f$ is a locally finite signed measure in $\Omega$ with $|f| \in\left(W_{0}^{1, p}(\Omega)\right)^{*}$ such that $(2.5)$ holds for all $\varphi \in C_{c}^{\infty}(\Omega)$, with $\lambda \in\left(0, \gamma_{0}^{1-p} \alpha_{0}(p-1)^{p-1}\right)$. Then for each $k>0$, there exists a solution $u_{k} \in W_{0}^{1, p}(\Omega)$ to (4.2) such that $e^{\frac{\delta\left|u_{k}\right|}{p-1}}-1 \in W_{0}^{1, p}(\Omega)$ for all $\delta \in\left[\gamma_{0}, \delta_{0}\right)$, with $\delta_{0}=(p-1)\left(\alpha_{0} / \lambda\right)^{\frac{1}{p-1}}$, and

$$
\left\|u_{k}\right\|_{W_{0}^{1, p}(\Omega)}+\left\|e^{\frac{\delta\left|u_{k}\right|}{p-1}}-1\right\|_{W_{0}^{1, p}(\Omega)} \leq M_{\delta} .
$$

Moreover, for any $\delta_{1}>\gamma_{0}$ such that (2.7) holds then we have

$$
\left\|e^{\frac{\delta_{1}\left|u_{k}\right|}{p-1}}-1\right\|_{W_{0}^{1, p}(\Omega)} \leq M_{\delta_{1}}
$$

Here the constants $M_{\delta}$ and $M_{\delta_{1}}$ are independent of $k$.

Proof. Since $\sigma \in\left(W_{0}^{1, p}(\Omega)\right)^{*}$ and $\left|\mathcal{H}_{k}(x, s, \xi)\right| \leq k$, by the theory of pseudomonotone operators (see, e.g., [26], [27, Chapter 6], and [8]), for any $\varepsilon>0$ there exists a solution $u_{k, \varepsilon} \in W_{0}^{1, p}(\Omega)$ to the equation

$$
-\operatorname{div} \mathcal{A}(x, u, \nabla u)+\varepsilon|u|^{p-2} u=\mathcal{H}_{k}(x, u, \nabla u)+\sigma \quad \text { in } \quad \Omega .
$$

The next step is to obtain uniform bounds of the form (4.3)-(4.4) for $\left\{u_{k, \varepsilon}\right\}$. However, we cannot directly apply Theorem 3.1 here since we do not know if $e^{\frac{\delta\left|u_{k, \varepsilon}\right|}{p-1}}-1 \in W_{0}^{1, p}(\Omega)$. The strategy here is to follow the proof of Theorem 3.1. For simplicity let us write $u=u_{k, \varepsilon}$, and for each $s>0$, we set $v_{s}=e^{\delta\left|u_{s}\right|} w_{s}$, where $u_{s}=T_{s}(u)$ and $w_{s}=\operatorname{sign}(u)\left[e^{\mu\left|u_{s}\right|}-1\right] / \mu$ with $\mu=\delta /(p-1)$. Then using $v_{s}$ as a test function for (4.5) we obtain the following equality

$$
I_{1}+I_{2}=I_{3}+I_{4}^{\prime}+I_{5}+I_{6}
$$

where the expressions for $I_{1}, I_{2}, I_{3}, I_{5}, I_{6}$ are as in the proof of Theorem 3.1. The term $I_{4}^{\prime}$ is similar to $I_{4}$ given in the proof of Theorem 3.1 except that $\mathcal{B}(x, u, \nabla u)$ is now replaced by $\mathcal{H}_{k}(x, u, \nabla u)$. That is,

$$
I_{4}^{\prime}:=\int_{\Omega} \mathcal{H}_{k}(x, u, \nabla u) e^{\delta\left|u_{s}\right|} w_{s} d x
$$


Thus lower estimates for $I_{1}, I_{2}$ and upper estimates for $I_{5}+I_{6}$ are unchanged; see (3.6), (3.7), and (3.12). As in (3.8) we have the following upper estimate for $I_{3}+I_{4}^{\prime}$ :

$$
\begin{aligned}
I_{3}+I_{4}^{\prime} \leq & \int_{\Omega}\left(-\delta+\gamma_{0}\right) \alpha_{0}\left|w_{s}\right| e^{\delta\left|u_{s}\right|}\left|\nabla u_{s}\right|^{p} d x \\
& +\int_{\Omega} \mathcal{H}_{k}(x, u, \nabla u) \operatorname{sign}(u) e^{\delta\left|u_{s}\right|}\left|w_{s}\right| \chi_{\{|u|>s\}} d x \\
\leq & \int_{\Omega} \mathcal{H}_{k}(x, u, \nabla u) \operatorname{sign}(u) e^{\delta\left|u_{s}\right|}\left|w_{s}\right| \chi_{\{|u|>s\}} d x .
\end{aligned}
$$

Thus, instead of (3.10), we now get

$$
I_{3}+I_{4}^{\prime} \leq k \int_{\Omega} e^{\delta s} \frac{e^{\mu s}-1}{\mu} \chi_{\{|u|>s\}} d x
$$

Similarly, instead of (3.14), we now obtain

$$
\begin{aligned}
I_{3}+I_{4}^{\prime} \leq & \int_{\Omega}(1-\varepsilon)\left(-\delta_{1}+\gamma_{0}\right) \frac{\alpha_{0}}{\mu_{1}}\left|\nabla w_{s}\right|^{p} d x+C(\varepsilon) \int_{\Omega}\left|\nabla u_{s}\right|^{p} d x \\
& +k \int_{\Omega} e^{\delta s} \frac{e^{\mu s}-1}{\mu} \chi_{\{|u|>s\}} d x .
\end{aligned}
$$

We recall that in (4.8), $\mu_{1}=\frac{\delta_{1}}{p-1}$ with $w, w_{s}$ to be understood as in (3.13).

When $\varepsilon>0$ and $s$ is such that $\varepsilon s^{p-1} \geq k$ by (3.7) and (4.7) we have

$$
I_{3}+I_{4}^{\prime}-I_{2} \leq 0
$$

and thus it follows from (4.6) that

$$
I_{1} \leq I_{5}+I_{6}
$$

With this, employing (3.6) and (3.12) we find

$$
\left\|\nabla w_{s}\right\|_{L^{p}(\Omega)} \leq C(\delta, \lambda, p)\left(\|F\|_{L^{\frac{p}{p-1}(\Omega)}}+\||f|\|_{\left(W_{0}^{1, p}(\Omega)\right)^{*}}\right)^{\frac{1}{p-1}} .
$$

At this point we let $s \nearrow_{\infty}$ to obtain that any solution $u=u_{k, \varepsilon}$ to (4.5) satisfies the bound

$$
\begin{aligned}
\left\|u_{k, \varepsilon}\right\|_{W_{0}^{1, p}(\Omega)}+\left\|e^{\frac{\delta\left|u_{k, \varepsilon}\right|}{p-1}}-1\right\|_{W_{0}^{1, p}(\Omega)} \leq \\
\quad \leq C(\delta, \lambda, p)\left(\|F\|_{L^{\frac{p}{p-1}(\Omega)}}+\||f|\|_{\left(W_{0}^{1, p}(\Omega)\right)^{*}}\right)^{\frac{1}{p-1}}
\end{aligned}
$$

for every $\delta \in\left[\gamma_{0}, \delta_{0}\right)$. 
For $\delta_{1}>\gamma_{0}$ such that (2.7) holds, using (4.8) and arguing similarly we obtain

$$
\begin{aligned}
\left\|e^{\frac{\delta_{1}\left|u_{k, \varepsilon}\right|}{p-1}}-1\right\|_{W_{0}^{1, p}(\Omega)} \leq & C\left(\|F\|_{L^{\frac{p}{p-1}(\Omega)}}+\||f|\|_{\left(W_{0}^{1, p}(\Omega)\right)^{*}}\right)^{\frac{1}{p-1}} \\
& +C \int_{\Omega}\left|\nabla u_{k, \varepsilon}\right|^{p} d x \\
\leq & C\left(\|F\|_{L^{\frac{p}{p-1}}(\Omega)}+\||f|\|_{\left(W_{0}^{1, p}(\Omega)\right)^{*}}\right)^{\frac{1}{p-1}} .
\end{aligned}
$$

As the bound (4.10) is uniform in $\varepsilon$, we can extract a subsequence, still denoted by $\varepsilon$, such that

$$
u_{k, \varepsilon} \rightarrow u_{k} \text { weakly in } W_{0}^{1, p}(\Omega) \text {, strongly in } L^{p}(\Omega) \text {, and a.e. in } \Omega \text {, }
$$

as $\varepsilon \searrow 0^{+}$for a function $u_{k} \in W_{0}^{1, p}(\Omega)$. Due to the pointwise a.e. convergence, we see that $u_{k}$ also satisfies (4.10)-(4.11) for every $\delta \in\left[\gamma_{0}, \delta_{0}\right)$ and every $\delta_{1}>\gamma_{0}$ such that $(2.7)$ holds.

Recall that we have

$$
-\operatorname{div} \mathcal{A}\left(x, u_{k, \varepsilon}, \nabla u_{k, \varepsilon}\right)+\varepsilon\left|u_{k, \varepsilon}\right|^{p-2} u_{k, \varepsilon}=\mathcal{H}_{k}\left(x, u_{k, \varepsilon}, \nabla u_{k, \varepsilon}\right)+\sigma \quad \text { in } \mathcal{D}^{\prime}(\Omega) .
$$

For each fixed $k>0$, we know $\mathcal{H}_{k}\left(x, u_{k, \varepsilon}, \nabla u_{k, \varepsilon}\right)-\varepsilon\left|u_{k, \varepsilon}\right|^{p-2} u_{k, \varepsilon}$ is uniformly bounded in $\varepsilon \in(0,1)$ as finite measures in $\Omega$. Thus by a convergence result shown in [6, Eqn (2.26)], we may further assume that

$$
\nabla u_{k, \varepsilon} \rightarrow \nabla u_{k} \quad \text { a.e. in } \Omega, \text { as } \varepsilon \searrow 0^{+}
$$

This allows us to pass to the limit in (4.12) as $\varepsilon \searrow 0^{+}$to see that $u_{k}$ solves (4.2) and satisfies the bounds (4.3)-(4.4).

\section{Proof of TheOrem 2.3}

This section is devoted to the proof of Theorem 2.3.

Proof. For each $k>0$, let $u_{k}$ be a solution of the approximate equation (4.2) as obtained in Proposition 4.1. Recall that $\mathcal{H}_{k}(x, s, \xi)$ is defined in (4.1). By (4.3)-(4.4) and Rellich's compactness theorem, there is a subsequence, still denoted by $k$, such that

$$
u_{k} \stackrel{k}{\rightarrow} u \text { weakly in } W_{0}^{1, p}(\Omega), \text { strongly in } L^{p}(\Omega), \text { and a.e. in } \Omega,
$$

for some function $u \in W_{0}^{1, p}(\Omega)$ such that $e^{\frac{\delta|u|}{p-1}}-1 \in W_{0}^{1, p}(\Omega)$ for each $\delta \in\left[\gamma_{0}, \delta_{0}\right)$, and $e^{\frac{\delta_{1}|u|}{p-1}}-1 \in W_{0}^{1, p}(\Omega)$ for any $\delta_{1}>\gamma_{0}$ such that $(2.7)$ holds. 
As $u_{k}$ solves (4.2), we have

$$
-\operatorname{div} \mathcal{A}\left(x, u_{k}, \nabla u_{k}\right)=\mathcal{H}_{k}\left(x, u_{k}, \nabla u_{k}\right)+\sigma \quad \text { in } \quad \Omega .
$$

Thus to show that $u$ is a solution of (2.6) it is enough to show that

$$
u_{k} \rightarrow u \text { strongly in } W_{0}^{1, p}(\Omega) \text { as } k \nearrow \infty,
$$

so that we can pass to the limit in (5.1) using (2.4), (4.3), and Vitali's Convergence Theorem.

For each $s>0$ we can write

$$
\nabla u_{k}-\nabla u=\nabla T_{s}\left(u_{k}\right)-\nabla T_{s}(u)+\nabla G_{s}\left(u_{k}\right)-\nabla G_{s}(u),
$$

where

$$
G_{s}(r):=r-T_{s}(r), \quad r \in \mathbb{R} .
$$

In order to show (5.2) we shall show that the following limits hold:

$$
\begin{gathered}
\lim _{s \rightarrow \infty} \sup _{k>0}\left\|\nabla G_{s}\left(u_{k}\right)-\nabla G_{s}(u)\right\|_{L^{p}(\Omega)}=0 \\
\lim _{k \rightarrow \infty}\left\|\nabla T_{s}\left(u_{k}\right)-\nabla T_{s}(u)\right\|_{L^{p}(\Omega)}=0 \quad \text { for each } s>0 .
\end{gathered}
$$

The rest of the proof will be devoted to the verification of these limits.

Proof of (5.3). Define $w_{k}=\left[e^{\frac{\delta}{p-1}\left|u_{k}\right|}-1\right] \frac{p-1}{\delta}$ and hence we get

$$
\begin{aligned}
\int_{\Omega}\left|\nabla G_{s}\left(u_{k}\right)\right|^{p} d x & =\int_{\left\{\left|u_{k}\right|>s\right\}}\left|\nabla u_{k}\right|^{p} d x \\
& =\int_{\left\{\left|u_{k}\right|>s\right\}} e^{-\frac{\delta p}{p-1}\left|u_{k}\right|}\left|\nabla w_{k}\right|^{p} d x \\
& \leq e^{-\frac{\delta p}{p-1} s} \int_{\left\{\left|u_{k}\right|>s\right\}}\left|\nabla w_{k}\right|^{p} d x
\end{aligned}
$$

Using the estimate (4.3), we then find

$$
\int_{\Omega}\left|\nabla G_{s}\left(u_{k}\right)\right|^{p} d x \leq C(\delta) e^{-\frac{\delta p}{p-1} s},
$$

which yields (5.3).

Proof of (5.4). Following [16] (see also the earlier works $[15,4]$ ), we shall make use of the following test function in (5.1):

$$
v_{k}=e^{\delta\left|T_{j}\left(u_{k}\right)\right|} \psi\left(z_{k}\right), \quad \text { with } j \geq s,
$$

where $z_{k}=T_{s}\left(u_{k}\right)-T_{s}(u)$ and $\psi$ is a $C^{1}$ and increasing function from $\mathbb{R}$ to $\mathbb{R}$ satisfying

$$
\psi(0)=0 \quad \text { and } \quad \psi^{\prime}-H_{0}|\psi| \geq 1
$$


where $H_{0}=\frac{b_{0}+\left(a_{0}+a_{1}\right) \delta}{\alpha_{0}}$. For example, the function $\psi(r)=2 r e^{\frac{H_{0}^{2} r^{2}}{4}}$ will do. We then have

$$
\begin{aligned}
& \int_{\Omega} \mathcal{A}\left(x, u_{k}, \nabla u_{k}\right) \cdot e^{\delta\left|T_{j}\left(u_{k}\right)\right|} \psi^{\prime}\left(z_{k}\right) \nabla z_{k} d x \\
& =\int_{\Omega}\left[\mathcal{H}_{k}\left(x, u_{k}, \nabla u_{k}\right)-\delta \mathcal{A}\left(x, \nabla u_{k}\right) \cdot \nabla T_{j}\left(u_{k}\right) \operatorname{sign}\left(u_{k}\right)\right] e^{\delta\left|T_{j}\left(u_{k}\right)\right|} \psi\left(z_{k}\right) d x \\
& \quad+\left\langle\sigma, e^{\delta\left|T_{j}\left(u_{k}\right)\right|} \psi\left(z_{k}\right)\right\rangle .
\end{aligned}
$$

Note that the term on the left-hand side in the above equality can be written as

$$
\begin{aligned}
& \int_{\Omega} \mathcal{A}\left(x, u_{k}, \nabla u_{k}\right) \cdot\left(\nabla T_{s}\left(u_{k}\right)-\nabla T_{s}(u)\right) e^{\delta\left|T_{j}\left(u_{k}\right)\right|} \psi^{\prime}\left(z_{k}\right) d x \\
& =\int_{\left\{\left|u_{k}\right| \leq s\right\}}\left(\mathcal{A}\left(x, T_{s}\left(u_{k}\right), \nabla T_{s}\left(u_{k}\right)\right)-\mathcal{A}\left(x, T_{s}\left(u_{k}\right), \nabla T_{s}(u)\right)\right) \cdot \\
& \quad \cdot\left(\nabla T_{s}\left(u_{k}\right)-\nabla T_{s}(u)\right) e^{\delta\left|T_{j}\left(u_{k}\right)\right|} \psi^{\prime}\left(z_{k}\right) d x \\
& \quad+\int_{\left\{\left|u_{k}\right| \leq s\right\}} \mathcal{A}\left(x, T_{s}\left(u_{k}\right), \nabla T_{s}(u)\right) \cdot\left(\nabla T_{s}\left(u_{k}\right)-\nabla T_{s}(u)\right) e^{\delta\left|T_{j}\left(u_{k}\right)\right|} \psi^{\prime}\left(z_{k}\right) d x \\
& \quad+\int_{\left\{\left|u_{k}\right|>s\right\}} \mathcal{A}\left(x, u_{k}, \nabla u_{k}\right) \cdot\left(-\nabla T_{s}(u)\right) e^{\delta\left|T_{j}\left(u_{k}\right)\right|} \psi^{\prime}\left(z_{k}\right) d x .
\end{aligned}
$$

Thus combining the last two equalities we obtain

$$
I_{1}=-I_{2}-I_{3}+I_{4}+I_{5}
$$

where we have defined

$$
\begin{gathered}
I_{1}=\int_{\left\{\left|u_{k}\right| \leq s\right\}}\left(\mathcal{A}\left(x, T_{s}\left(u_{k}\right), \nabla T_{s}\left(u_{k}\right)\right)-\mathcal{A}\left(x, T_{s}\left(u_{k}\right), \nabla T_{s}(u)\right)\right) \cdot \\
\cdot\left(\nabla T_{s}\left(u_{k}\right)-\nabla T_{s}(u)\right) e^{\delta\left|T_{j}\left(u_{k}\right)\right|} \psi^{\prime}\left(z_{k}\right) d x \\
I_{2}=\int_{\left\{\left|u_{k}\right| \leq s\right\}} \mathcal{A}\left(x, T_{s}\left(u_{k}\right), \nabla T_{s}(u)\right) \cdot\left(\nabla T_{s}\left(u_{k}\right)-\nabla T_{s}(u)\right) e^{\delta\left|T_{j}\left(u_{k}\right)\right|} \psi^{\prime}\left(z_{k}\right) d x, \\
I_{3}=\int_{\left\{\left|u_{k}\right|>s\right\}} \mathcal{A}\left(x, u_{k}, \nabla u_{k}\right) \cdot\left(-\nabla T_{s}(u)\right) e^{\delta\left|T_{j}\left(u_{k}\right)\right|} \psi^{\prime}\left(z_{k}\right) d x, \\
I_{4}=\int_{\Omega}\left[\mathcal{H}_{k}\left(x, u_{k}, \nabla u_{k}\right)-\delta \mathcal{A}\left(x, u_{k}, \nabla u_{k}\right) \cdot \nabla T_{j}\left(u_{k}\right) \operatorname{sign}\left(u_{k}\right)\right] e^{\delta\left|T_{j}\left(u_{k}\right)\right|} \psi\left(z_{k}\right) d x,
\end{gathered}
$$

and

$$
I_{5}=\left\langle\sigma, e^{\delta\left|T_{j}\left(u_{k}\right)\right|} \psi\left(z_{k}\right)\right\rangle
$$

We now write $I_{4}$ as

$$
I_{4}=I_{4}^{\prime}+I_{4}^{\prime \prime}
$$


where

$$
\begin{aligned}
I_{4}^{\prime} & :=\int_{\left\{\left|u_{k}\right|>s\right\}} H_{k, j}(x) e^{\delta\left|T_{j}\left(u_{k}\right)\right|} \psi\left(z_{k}\right) d x, \\
I_{4}^{\prime \prime} & :=\int_{\left\{\left|u_{k}\right| \leq s\right\}} H_{k, j}(x) e^{\delta\left|T_{j}\left(u_{k}\right)\right|} \psi\left(z_{k}\right) d x,
\end{aligned}
$$

with

$$
H_{k, j}(x):=\mathcal{H}_{k}\left(x, u_{k}, \nabla u_{k}\right)-\delta \mathcal{A}\left(x, u_{k}, \nabla u_{k}\right) \cdot \nabla T_{j}\left(u_{k}\right) \operatorname{sign}\left(u_{k}\right) .
$$

Note that $\left|\nabla T_{j}\left(u_{k}\right)\right| \leq\left|\nabla u_{k}\right|$ and hence using the growth conditions in (2.3) and (2.4) we get

$$
\begin{aligned}
\left|I_{4}^{\prime \prime}\right| \leq & \int_{\left\{\left|u_{k}\right| \leq s\right\}}\left(b_{0}\left|\nabla u_{k}\right|^{p}+b_{1}\left|u_{k}\right|^{m}+\delta a_{0}\left|\nabla u_{k}\right|^{p}+\delta a_{1}\left|u_{k}\right|^{p-1}\left|\nabla u_{k}\right|\right) \times \\
\times e^{\delta\left|T_{j}\left(u_{k}\right)\right|}\left|\psi\left(z_{k}\right)\right| d x & \int_{\left\{\left|u_{k}\right| \leq s\right\}}\left(b_{0}+\delta\left(a_{0}+a_{1}\right)\right)\left|\nabla u_{k}\right|^{p} e^{\delta\left|T_{j}\left(u_{k}\right)\right|}\left|\psi\left(z_{k}\right)\right| d x \\
& +\int_{\left\{\left|u_{k}\right| \leq s\right\}}\left(b_{1}\left|u_{k}\right|^{m}+c(p) \delta a_{1}\left|u_{k}\right|^{p}\right) e^{\delta\left|T_{j}\left(u_{k}\right)\right|}\left|\psi\left(z_{k}\right)\right| d x \\
\leq & \frac{b_{0}+\delta\left(a_{0}+a_{1}\right)}{\alpha_{0}} \int_{\left\{\left|u_{k}\right| \leq s\right\}} \mathcal{A}\left(x, T_{s}\left(u_{k}\right), \nabla T_{s}\left(u_{k}\right)\right) \cdot \nabla T_{s}\left(u_{k}\right) e^{\delta\left|T_{j}\left(u_{k}\right)\right|}\left|\psi\left(z_{k}\right)\right| d x \\
& +\int_{\left\{\left|u_{k}\right| \leq s\right\}}\left(b_{1}\left|u_{k}\right|^{m}+c(p) \delta a_{1}\left|u_{k}\right|^{p}\right) e^{\delta\left|T_{j}\left(u_{k}\right)\right|}\left|\psi\left(z_{k}\right)\right| d x,
\end{aligned}
$$

where we used Young's inequality in the second inequality and the coercivity condition (2.2) in the last inequality. Thus, recalling that $H_{0}=\frac{b_{0}+\delta\left(a_{0}+a_{1}\right)}{\alpha_{0}}$, we find

$$
\begin{aligned}
\left|I_{4}^{\prime \prime}\right| \leq & H_{0} \int_{\left\{\left|u_{k}\right| \leq s\right\}}\left[\mathcal{A}\left(x, T_{s}\left(u_{k}\right), \nabla T_{s}\left(u_{k}\right)\right)-\mathcal{A}\left(x, T_{s}\left(u_{k}\right), \nabla T_{s}(u)\right)\right] \cdot \\
& +H_{0} \int_{\left\{\left|u_{k}\right| \leq s\right\}} \mathcal{A}\left(x, T_{s}\left(u_{k}\right)-\nabla T_{s}(u)\right] e^{\delta\left|T_{j}\left(u_{k}\right)\right|}\left|\psi\left(z_{k}\right)\right| d x \\
& +H_{0} \int_{\left\{\left|u_{k}\right| \leq s\right\}} \mathcal{A}\left(x, T_{s}\left(u_{k}\right), \nabla T_{s}\left(u_{k}\right)\right) \cdot\left[\nabla T_{s}\left(u_{k}\right)-\nabla T_{s}(u)\right] e^{\delta\left|T_{j}\left(u_{k}\right)\right|}\left|\psi\left(z_{k}\right)\right| d x \\
& +\int_{\left\{\left|u_{k}\right| \leq s\right\}}\left(b_{1}\left|u_{k}\right|^{m}+c(p) \delta a_{1}\left|u_{k}\right|^{p-1}\left|\nabla T_{s}\left(u_{k}\right)\right|\right) e^{\delta\left|T_{j}\left(u_{k}\right)\right|}\left|\psi\left(z_{k}\right)\right| d x
\end{aligned}
$$

Using this bound, equalities (5.7)-(5.8), and the inequality in (5.6), we now obtain

$$
I_{1}^{\prime} \leq-I_{2}-I_{3}+I_{4}^{\prime}+I_{5}+I_{6}+I_{7}+I_{8}
$$

where

$$
I_{1}^{\prime}=\int_{\left\{\left|u_{k}\right| \leq s\right\}}\left(\mathcal{A}\left(x, T_{s}\left(u_{k}\right), \nabla T_{s}\left(u_{k}\right)\right)-\mathcal{A}\left(x, T_{s}(u), \nabla T_{s}(u)\right)\right) \cdot\left(\nabla T_{s}\left(u_{k}\right)-\nabla T_{s}(u)\right) d x
$$




$$
\begin{gathered}
I_{6}=H_{0} \int_{\left\{\left|u_{k}\right| \leq s\right\}} \mathcal{A}\left(x, T_{s}\left(u_{k}\right), \nabla T_{s}(u)\right) \cdot\left[\nabla T_{s}\left(u_{k}\right)-\nabla T_{s}(u)\right] e^{\delta\left|T_{j}\left(u_{k}\right)\right|}\left|\psi\left(z_{k}\right)\right| d x, \\
I_{7}=H_{0} \int_{\left\{\left|u_{k}\right| \leq s\right\}} \mathcal{A}\left(x, T_{s}\left(u_{k}\right), \nabla T_{s}\left(u_{k}\right)\right) \cdot \nabla T_{s}(u) e^{\delta\left|T_{j}\left(u_{k}\right)\right|}\left|\psi\left(z_{k}\right)\right| d x
\end{gathered}
$$

and

$$
I_{8}=\int_{\left\{\left|u_{k}\right| \leq s\right\}}\left(b_{1}\left|u_{k}\right|^{m}+c(p) \delta a_{1}\left|u_{k}\right|^{p-1}\left|\nabla T_{s}\left(u_{k}\right)\right|\right) e^{\delta\left|T_{j}\left(u_{k}\right)\right|}\left|\psi\left(z_{k}\right)\right| d x .
$$

We shall next treat each term on the right-hand side of (5.9).

The term $I_{2}$ : We know that $u_{k} \stackrel{k}{\rightarrow} u$ a.e., from which we see that $z_{k} \stackrel{k}{\rightarrow} 0$ a.e. and hence

$$
\mathcal{A}\left(x, T_{s}\left(u_{k}\right), \nabla T_{s}(u)\right) e^{\delta\left|T_{j}\left(u_{k}\right)\right|} \psi^{\prime}\left(z_{k}\right) \stackrel{k}{\rightarrow} \mathcal{A}\left(x, T_{s}(u), \nabla T_{s}(u)\right) e^{\delta\left|T_{j}(u)\right|} \psi^{\prime}(0) \quad \text { a.e. }
$$

Thus using the pointwise estimate, which follows from (2.3),

$$
\left|\mathcal{A}\left(x, T_{s}\left(u_{k}\right), \nabla T_{s}(u)\right) e^{\delta\left|T_{j}\left(u_{k}\right)\right|} \psi^{\prime}\left(z_{k}\right)\right| \leq e^{\delta j} \max _{r \in[-2 s, 2 s]}\left|\psi^{\prime}(r)\right|\left[a_{0}\left|\nabla T_{s}(u)\right|^{p-1}+a_{1} s^{p-1}\right]
$$

and the fact that $\left|\nabla T_{s}(u)\right|^{p-1} \in L^{\frac{p}{p-1}}(\Omega)$, it follows from Lebesgue's Dominated Convergence Theorem that

$$
\mathcal{A}\left(x, T_{s}\left(u_{k}\right), \nabla T_{s}(u)\right) e^{\delta\left|T_{j}\left(u_{k}\right)\right|} \psi^{\prime}\left(z_{k}\right) \stackrel{k}{\rightarrow} \mathcal{A}\left(x, T_{s}(u), \nabla T_{s}(u)\right) e^{\delta\left|T_{j}(u)\right|} \psi^{\prime}(0)
$$

strongly in $L^{\frac{p}{p-1}}\left(\Omega, \mathbb{R}^{n}\right)$.

Since $\left\|T_{s}\left(u_{k}\right)\right\|_{W_{0}^{1, p}(\Omega)}$ is uniformly bounded in $k$ and $T_{s}\left(u_{k}\right) \stackrel{k}{\rightarrow} T_{s}(u)$ a.e. we get that $\nabla T_{s}\left(u_{k}\right) \stackrel{k}{\rightarrow} \nabla T_{s}(u)$ weakly in $L^{p}\left(\Omega, \mathbb{R}^{n}\right)$. Also, since

$$
\chi_{\left\{\left|u_{k}\right| \leq s\right\}} \stackrel{k}{\rightarrow} \chi_{\{|u| \leq s\}} \text { a.e. in } \Omega \backslash\{|u|=s\} \text { while }\left|\nabla T_{s}(u)\right|=0 \text { a.e. on }\{|u|=s\},
$$

we have from Lebesgue's Dominated Convergence Theorem that

$$
\nabla T_{s}(u) \chi_{\left\{\left|u_{k}\right| \leq s\right\}} \stackrel{k}{\rightarrow} \nabla T_{s}(u) \chi_{\{|u| \leq s\}}=\nabla T_{s}(u) \quad \text { strongly in } \quad L^{p}\left(\Omega, \mathbb{R}^{n}\right) .
$$

Thus with the observation $\chi_{\left\{\left|u_{k}\right| \leq s\right\}}\left(\nabla T_{s}\left(u_{k}\right)-\nabla T_{s}(u)\right)=\nabla T_{s}\left(u_{k}\right)-\nabla T_{s}(u) \chi_{\left\{\left|u_{k}\right| \leq s\right\}}$, we see that

$$
\chi_{\left\{\left|u_{k}\right| \leq s\right\}}\left(\nabla T_{s}\left(u_{k}\right)-\nabla T_{s}(u)\right) \stackrel{k}{\rightarrow} 0 \quad \text { weakly in } \quad L^{p}\left(\Omega, \mathbb{R}^{n}\right) .
$$

The above calculations imply that $\lim _{k \rightarrow \infty} I_{2}=0$.

The term $I_{3}$ : By $(2.3),\left|\mathcal{A}\left(x, u_{k}, \nabla u_{k}\right)\right|$ is uniformly bounded in $L^{\frac{p}{p-1}}(\Omega)$. On the other hand, again by (5.10) and Lebesgue's Dominated Convergence Theorem we have

$$
\left|\chi_{\left\{\left|u_{k}\right|>s\right\}}\left(-\nabla T_{s}(u)\right) e^{\delta\left|T_{j}\left(u_{k}\right)\right|} \psi^{\prime}\left(z_{k}\right)\right| \stackrel{k}{\rightarrow} 0 \quad \text { strongly in } \quad L^{p}(\Omega) .
$$

Thus we see that $\lim _{k \rightarrow \infty} I_{3}=0$. 
The term $I_{4}^{\prime}$ : We have the inequalities $\mathcal{A}\left(x, u_{k}, \nabla u_{k}\right) \cdot \nabla T_{j}\left(u_{k}\right) \geq \alpha_{0}\left|\nabla u_{k}\right|^{p} \chi_{\left\{\left|u_{k}\right| \leq j\right\}}$ and $\chi_{\left\{\left|u_{k}\right|>s\right\}} \operatorname{sign}\left(u_{k}\right) \psi\left(z_{k}\right) \geq 0$. Thus using the second inequality in (2.4) we see that

$$
\begin{aligned}
I_{4}^{\prime} & =\int_{\left\{\left|u_{k}\right|>s\right\}}\left[\operatorname{sign}\left(u_{k}\right) \mathcal{H}_{k}\left(x, u_{k}, \nabla u_{k}\right)-\delta \mathcal{A}\left(x, u_{k}, \nabla u_{k}\right) \cdot \nabla T_{j}\left(u_{k}\right)\right] \times \\
& \times \operatorname{sign}\left(u_{k}\right) e^{\delta\left|T_{j}\left(u_{k}\right)\right|} \psi\left(z_{k}\right) d x \\
\leq & \int_{\left\{\left|u_{k}\right|>s\right\}}\left[\gamma_{0} \alpha_{0}\left|\nabla u_{k}\right|^{p}-\delta \alpha_{0}\left|\nabla u_{k}\right|^{p} \chi_{\left\{\left|u_{k}\right| \leq j\right\}}\right] \operatorname{sign}\left(u_{k}\right) e^{\delta\left|T_{j}\left(u_{k}\right)\right|} \psi\left(z_{k}\right) d x \\
\leq & \int_{\left\{\left|u_{k}\right|>j\right\}} \gamma_{0} \alpha_{0}\left|\nabla u_{k}\right|^{p} \operatorname{sign}\left(u_{k}\right) e^{\delta\left|T_{j}\left(u_{k}\right)\right|} \psi\left(z_{k}\right) d x,
\end{aligned}
$$

where we used that $\delta \geq \gamma_{0}$ and $j \geq s$ in the last inequality. At this point, using (5.5) with $j$ in place of $s$, we get

$$
\begin{aligned}
I_{4}^{\prime} & \leq \gamma_{0} \alpha_{0} \max _{r \in[-2 s, 2 s]}|\psi(r)| e^{\delta j} \int_{\left\{\left|u_{k}\right|>j\right\}}\left|\nabla u_{k}\right|^{p} d x \\
& \leq C(\delta) \gamma_{0} \alpha_{0} \max _{r \in[-2 s, 2 s]}|\psi(r)| e^{\delta j} e^{-\frac{\delta p}{p-1} j} .
\end{aligned}
$$

This yields that $\lim \sup _{j \rightarrow \infty} \sup _{k>0} I_{4}^{\prime}=0$.

The term $I_{5}$ : Since $f \in\left(W_{0}^{1, p}(\Omega)\right)^{*}$, there is a vector field $F_{1} \in L^{\frac{p}{p-1}}\left(\Omega, \mathbb{R}^{n}\right)$ such that $\operatorname{div} F_{1}=f$ in $\mathcal{D}^{\prime}(\Omega)$. Thus $\sigma=\operatorname{div}\left(F+F_{1}\right)$ which yields

$$
\begin{aligned}
I_{5}= & \delta \int_{\Omega}\left(F+F_{1}\right) \cdot e^{\delta\left|T_{j}\left(u_{k}\right)\right|} \psi\left(z_{k}\right) \nabla T_{j}\left(u_{k}\right) \operatorname{sign}\left(u_{k}\right) d x \\
& +\int_{\Omega}\left(F+F_{1}\right) \cdot e^{\delta\left|T_{j}\left(u_{k}\right)\right|} \psi^{\prime}\left(z_{k}\right) \nabla z_{k} d x .
\end{aligned}
$$

As $\psi(0)=0$ we have $\left(F+F_{1}\right) e^{\delta\left|T_{j}\left(u_{k}\right)\right|} \psi\left(z_{k}\right) \stackrel{k}{\rightarrow}(0, \ldots, 0)$ a.e. in $\Omega$. Thus by Lebesgue's Dominated Convergence Theorem we find

$$
\left(F+F_{1}\right) e^{\delta\left|T_{j}\left(u_{k}\right)\right|} \psi\left(z_{k}\right) \stackrel{k}{\rightarrow}(0, \ldots, 0) \quad \text { strongly in } L^{\frac{p}{p-1}}\left(\Omega, \mathbb{R}^{n}\right) .
$$

Since $\nabla T_{j}\left(u_{k}\right) \operatorname{sign}\left(u_{k}\right)$ is uniformly bounded in $L^{p}\left(\Omega, \mathbb{R}^{n}\right)$, we then conclude that

$$
\delta \int_{\Omega}\left(F+F_{1}\right) \cdot e^{\delta\left|T_{j}\left(u_{k}\right)\right|} \psi\left(z_{k}\right) \nabla T_{j}\left(u_{k}\right) \operatorname{sign}\left(u_{k}\right) d x \stackrel{k}{\rightarrow} 0 .
$$

We now write

$$
\int_{\Omega}\left(F+F_{1}\right) \cdot e^{\delta\left|T_{j}\left(u_{k}\right)\right|} \psi^{\prime}\left(z_{k}\right) \nabla z_{k} d x=R_{1}+R_{2}
$$


where

$$
\begin{aligned}
R_{1} & :=\int_{\left\{\left|u_{k}\right| \leq s\right\}}\left(F+F_{1}\right) \cdot e^{\delta\left|T_{j}\left(u_{k}\right)\right|} \psi^{\prime}\left(z_{k}\right) \nabla z_{k} d x \\
R_{2} & :=\int_{\left\{\left|u_{k}\right|>s\right\}}\left(F+F_{1}\right) \cdot e^{\delta\left|T_{j}\left(u_{k}\right)\right|} \psi^{\prime}\left(z_{k}\right) \nabla z_{k} d x .
\end{aligned}
$$

Again by Lebesgue's Dominated Convergence Theorem we have

$$
\left(F+F_{1}\right) e^{\delta\left|T_{j}\left(u_{k}\right)\right|} \psi^{\prime}\left(z_{k}\right) \stackrel{k}{\rightarrow}\left(F+F_{1}\right) e^{\delta\left|T_{j}(u)\right|} \psi^{\prime}(0) \quad \text { strongly in } \quad L^{\frac{p}{p-1}}\left(\Omega, \mathbb{R}^{n}\right) .
$$

Thus using (5.11) (recall that $\left.\nabla z_{k}=\nabla T_{s}\left(u_{k}\right)-\nabla T_{s}(u)\right)$ we obtain that

$$
R_{1} \stackrel{k}{\rightarrow} 0 \text {. }
$$

On the other hand, from the definition of $z_{k}$ we have

$$
R_{2}=\int_{\Omega}\left(F+F_{1}\right) \cdot e^{\delta\left|T_{j}\left(u_{k}\right)\right|} \psi^{\prime}\left(z_{k}\right)\left(-\nabla T_{s}(u)\right) \chi_{\left\{\left|u_{k}\right|>s\right\}} d x .
$$

Then by (5.10), Hölder's inequality, and Lebesgue's Dominated Convergence Theorem, it follows that

$$
R_{2} \stackrel{k}{\rightarrow} 0
$$

Now recalling (5.14) we get

$$
\int_{\Omega}\left(F+F_{1}\right) \cdot e^{\delta\left|T_{j}\left(u_{k}\right)\right|} \psi^{\prime}\left(z_{k}\right) \nabla z_{k} d x \stackrel{k}{\rightarrow} 0 .
$$

Hence using (5.13) and (5.15) in (5.12) we conclude that $\lim _{k \rightarrow \infty} I_{5}=0$.

The terms $I_{6}, I_{7}$, and $I_{8}$ : Since $\psi(0)=0$, by Lebesgue's Dominated Convergence Theorem we find

$$
\chi_{\left\{\left|u_{k}\right| \leq s\right\}} \mathcal{A}\left(x, T_{s}\left(u_{k}\right), \nabla T_{s}(u)\right) e^{\delta\left|T_{j}\left(u_{k}\right)\right|}\left|\psi\left(z_{k}\right)\right| \stackrel{k}{\rightarrow} 0 \quad \text { strongly in } \quad L^{\frac{p}{p-1}}\left(\Omega, \mathbb{R}^{n}\right)
$$

and

$$
\chi_{\left\{\left|u_{k}\right| \leq s\right\}} \nabla T_{s}(u) e^{\delta\left|T_{j}\left(u_{k}\right)\right|}\left|\psi\left(z_{k}\right)\right| \stackrel{k}{\rightarrow} 0 \quad \text { strongly in } \quad L^{p}\left(\Omega, \mathbb{R}^{n}\right) .
$$

On the other hand, $\nabla T_{s}\left(u_{k}\right)-\nabla T_{s}(u)$ and $\mathcal{A}\left(x, T_{s}\left(u_{k}\right), \nabla T_{s}\left(u_{k}\right)\right)$ are uniformly bounded in $L^{p}\left(\Omega, \mathbb{R}^{n}\right)$ and in $L^{\frac{p}{p-1}}\left(\Omega, \mathbb{R}^{n}\right)$, respectively. Thus we obtain that

$$
\lim _{k \rightarrow \infty} I_{6}=\lim _{k \rightarrow \infty} I_{7}=0
$$


As for the term $I_{8}$, we estimate

$$
I_{8} \leq \int_{\left\{\left|u_{k}\right| \leq s\right\}}\left(b_{1}|s|^{m}+c(p) \delta a_{1} s^{p-1}\right) e^{\delta s}\left|\psi\left(z_{k}\right)\right| d x
$$

which also converges to zero, as $k \nearrow \infty$, by Lebesgue's Dominated Convergence Theorem.

We have shown that $\lim _{k \rightarrow \infty}\left(-I_{2}-I_{3}+I_{5}+I_{6}+I_{7}+I_{8}\right)=0$ and $\lim \sup _{j \rightarrow \infty} \sup _{k>0} I_{4}^{\prime}=0$. For each fixed $s>0$, we now let

$$
D_{k}=\left(\mathcal{A}\left(x, T_{s}\left(u_{k}\right), \nabla T_{s}\left(u_{k}\right)\right)-\mathcal{A}\left(x, T_{s}\left(u_{k}\right), \nabla T_{s}(u)\right)\right) \cdot\left(\nabla T_{s}\left(u_{k}\right)-\nabla T_{s}(u)\right) .
$$

As $D_{k} \geq 0$ (by $\left.(2.1)\right)$, in view of $(5.9)$ we find that

$$
\int_{\left\{\left|u_{k}\right| \leq s\right\}} D_{k} d x \stackrel{k}{\rightarrow} 0 .
$$

On the other hand, by (5.10),

$$
\begin{aligned}
\chi_{\left\{\left|u_{k}\right|>s\right\}} D_{k} & =\chi_{\left\{\left|u_{k}\right|>s\right\}}\left[\mathcal{A}\left(x, T_{s}\left(u_{k}\right), 0\right)-\mathcal{A}\left(x, T_{s}\left(u_{k}\right), \nabla T_{s}(u)\right)\right] \cdot\left(-\nabla T_{s}(u)\right) \\
& \rightarrow 0 \quad \text { a.e. as } k \nearrow \infty .
\end{aligned}
$$

It then follows from Lebesgue's Dominated Convergence Theorem that

$$
\int_{\left\{\left|u_{k}\right|>s\right\}} D_{k} d x \stackrel{k}{\rightarrow} 0
$$

Combining (5.16)-(5.17) we obtain

$$
\int_{\Omega} D_{k} d x \stackrel{k}{\rightarrow} 0
$$

At this point we use the conditions (2.1)-(2.3) and a result of F. E. Browder (see [10] or [7, Lemma 5]) to complete the proof of (5.4).

\section{Proof of Theorems 1.2 And 1.8}

We are now ready to prove Theorem 1.2.

Proof of Theorem 1.2. (i) Suppose that (1.1) has a solution in $u \in W_{0}^{1, p}(\Omega)$ such that (1.5) holds for some $A>0$. Then letting $F=|\nabla u|^{p-2} \nabla u$, we immediately have the desired representation for $\sigma$.

(ii) Suppose that $\sigma=\operatorname{div} F+f$ where $F \in L^{\frac{p}{p-1}}\left(\Omega, \mathbb{R}^{n}\right)$ and $f$ is a locally finite signed measure in $\Omega$ with $|f| \in\left(W_{0}^{1, p}(\Omega)\right)^{*}$ such that (1.7) holds for some $\lambda \in\left(0,(p-1)^{p-1}\right)$. Applying Theorem 2.3 we obtain a solution to (1.1) that satisfies all of the properties 
stated in Theorem 1.2(ii) except the Poincaré-Sobolev inequality (1.5). To verify it, we use $|\varphi|^{p}, \varphi \in C_{c}^{\infty}(\Omega)$, as a test function in (1.1) to get

$$
\int_{\Omega}|\varphi|^{p}|\nabla u|^{p} d x=p \int_{\Omega}|\nabla u|^{p-2} \nabla u \cdot \nabla|\varphi||\varphi|^{p-1} d x+\left\langle\sigma,|\varphi|^{p}\right\rangle .
$$

Thus by Hölder's inequality and condition (1.7) we find

$$
\int_{\Omega}|\varphi|^{p}|\nabla u|^{p} d x \leq p\left(\int_{\Omega}|\nabla u|^{p}|\varphi|^{p} d x\right)^{\frac{p-1}{p}}\left(\int_{\Omega}|\nabla \varphi|^{p} d x\right)^{\frac{1}{p}}+(p-1)^{p-1} \int_{\Omega}|\nabla \varphi|^{p} d x .
$$

At this point applying Young's inequality we obtain the Poincaré-Sobolev inequality (1.5) with some $A=A(p)>0$.

Finally, we prove Theorem 1.8 .

Proof of Theorem 1.8. By Theorem 1.2(ii) we can find a solution $u \in W_{0}^{1, p}(\Omega)$ to (1.1) such that both $e^{u}-1$ and $e^{\frac{u}{p-1}}-1 \in W_{0}^{1, p}(\Omega)$. Thus if we define $v=e^{\frac{u}{p-1}}$ then it holds that $v-1 \in W_{0}^{1, p}(\Omega)$ and $v^{p-1}=e^{u} \in W^{1, p}(\Omega)$. We will show that $v$ is indeed a solution of (1.3).

We first observe that the function $e^{u}|\nabla u|^{p}$ belongs to $L^{1}(\Omega)$. Indeed,

$$
\begin{aligned}
\int_{\Omega} e^{u}|\nabla u|^{p} d x & =\int_{\{u \geq 0\} \cap \Omega} e^{u}|\nabla u|^{p} d x+\int_{\{u<0\} \cap \Omega} e^{u}|\nabla u|^{p} d x \\
& \leq \int_{\{u \geq 0\} \cap \Omega} e^{p u}|\nabla u|^{p} d x+\int_{\{u<0\} \cap \Omega}|\nabla u|^{p} d x \\
& \leq \int_{\Omega}\left|\nabla\left(e^{u}\right)\right|^{p} d x+\int_{\Omega}|\nabla u|^{p} d x<+\infty .
\end{aligned}
$$

Let $\varphi \in C_{c}^{\infty}(\Omega)$. Using $\phi_{j}:=\varphi \min \left\{e^{u}, j\right\}, j>0$, as a test function for (1.1) we have

$$
\int_{\Omega}|\nabla u|^{p-2} \nabla u \cdot \nabla \phi_{j} d x=\int_{\Omega}|\nabla u|^{p} \phi_{j} d x+\left\langle\sigma, \phi_{j}\right\rangle .
$$

We now send $j \nearrow \infty$ in $(6.1)$ to obtain

$$
\int_{\Omega}|\nabla u|^{p-2} \nabla u \cdot \nabla\left(\varphi e^{u}\right) d x=\int_{\Omega}|\nabla u|^{p} \varphi e^{u} d x+\left\langle\sigma, \varphi e^{u}\right\rangle .
$$

Here we use $e^{u}|\nabla u|^{p} \in L^{1}(\Omega)$ and Lebesgue's Dominated Convergence Theorem. We note that actually by Lemma 2.2 we can immediately use $\varphi e^{u}$ as a test function. Thus after expanding and simplifying we get

$$
\int_{\Omega}\left[|\nabla u|^{p-2} \nabla u \cdot \nabla \varphi\right] e^{u} d x=\left\langle\sigma, \varphi e^{u}\right\rangle=\left\langle\sigma, \varphi v^{p-1}\right\rangle .
$$

Note that $\nabla v=(p-1)^{-1} e^{\frac{u}{p-1}} \nabla u$ and thus $\nabla u=(p-1) e^{-\frac{u}{p-1}} \nabla v$. This yields that

$$
\left(|\nabla u|^{p-2} \nabla u\right) e^{u}=(p-1)^{p-1}|\nabla v|^{p-2} \nabla v,
$$


and hence

$$
\int_{\Omega}|\nabla v|^{p-2} \nabla v \cdot \nabla \varphi d x=(p-1)^{1-p}\left\langle\sigma, \varphi v^{p-1}\right\rangle
$$

for all $\varphi \in C_{c}^{\infty}(\Omega)$. This shows that $v$ is a solution of (1.3) as claimed.

Finally, inequality (1.10) follows from (1.5) and the equality $\left|\frac{\nabla v}{v}\right|^{p}=(p-1)^{-p}|\nabla u|^{p}$.

Remark 6.1. The above argument also works for the more general equation

$$
-\operatorname{div} \mathcal{A}(x, \nabla v)=(p-1)^{1-p} \sigma v^{p-1} \text { in } \Omega, \quad v \geq 0 \text { in } \Omega, \quad v=1 \text { on } \partial \Omega,
$$

where $\mathcal{A}(x, \xi)$ satisfies (2.1)-(2.3) with $0<\alpha_{0} \leq a_{0}$ and the homogeneity condition

$$
\mathcal{A}(x, t \xi)=t^{p-1} \mathcal{A}(x, \xi) \quad \text { for all } t>0 .
$$

In this case $v=e^{\frac{u}{p-1}}$, where $u \in W_{0}^{1, p}(\Omega)$ solves the equation

$$
-\operatorname{div} \mathcal{A}(x, \nabla u)=\mathcal{A}(x, \nabla u) \cdot \nabla u+\sigma .
$$

By Theorem 2.3, to guarantee that both $e^{u}-1$ and $e^{\frac{u}{p-1}}-1 \in W_{0}^{1, p}(\Omega)$, we also need to assume

$$
\lambda \in\left(0, a_{0}^{1-p} \alpha_{0}^{p}(p-1)^{p-1}\right) \quad \text { if } \quad \frac{a_{0}}{\alpha_{0}} \geq p-1
$$

and

$$
\lambda \in\left(0, \alpha_{0} p-a_{0}\right) \quad \text { if } \quad \frac{a_{0}}{\alpha_{0}}<p-1 .
$$

However, note that no regularity assumption in the $x$-variable of $\mathcal{A}(x, \xi)$ is needed here.

\section{REFERENCES}

[1] B. Abdellaoui, A. Dall'Aglio, and I. Peral, Some remarks on elliptic problems with critical growth in the gradient, J. Differential Equations 222 (2006) 21-62.

[2] D. R. Adams and L. I. Hedberg, Function Spaces and Potential Theory, Springer-Verlag, Berlin, 1996.

[3] A. Ancona, On strong barriers and an inequality of Hardy for domains in $\mathbb{R}^{n}$, J. London Math. Soc. 34 (1986), 274-290.

[4] A. Bensoussan, L. Boccardo, and F. Murat, On a nonlinear partial differential equation having natural growth terms and unbounded solution, Ann. Inst. H. Poincaré Anal. Non Linéaire, 5 (1988), 347-364.

[5] L. Boccardo, T. Gallouët, and L. Orsina, Existence and uniqueness of entropy solutions for nonlinear elliptic equations with measure data, Ann. Inst. H. Poincaré Anal. Non Linéaire 13 (1996) 539-551.

[6] L. Boccardo and F. Murat, Almost everywhere convergence of the gradients of solutions to elliptic and parabolic equations, Nonlinear Anal. 19 (1992), 581-597.

[7] L. Boccardo, F. Murat, and J.-P. Puel, Existence of bounded solutions for nonlinear elliptic unilateral problems, Ann. Mat. Pura Appl. (4) 152 (1988), 183-196. 
[8] H. Brézis, Équations et inéquations non-linéaires dans les espaces vectoriel en dualité, Ann. Inst. Fourier 18 (1968), 115-176.

[9] H. Brézis and F. E. Browder, Some properties of higher order Sobolev spaces, J. Math. Pures Appl. 61 (1982), 245-259.

[10] F. E. Browder, Existence theorems for nonlinear partial differential equations, in S.-S. Chern, S. Smale (Eds), Proc. Sympos. Pure Math., Vol. XVI, pp. 1-60, Amer. Math. Soc., Providence, R.I. 1970.

[11] D. M. Duc, N. C. Phuc, and T. V. Nguyen, Weighted Sobolev's inequalities for bounded domains and singular elliptic equations, Indiana Univ. Math. J. 56 (2007), 615-642.

[12] S.-Y. A. Chang, J. M. Wilson, and T. H. Wolff, Some weighted norm inequalities concerning the Schrödinger operators, Comment. Math. Helv. 60 (1985), 217-246.

[13] C. Fefferman, The uncertainty principle, Bull. Amer. Math. Soc. 9 (1983), 129-206.

[14] V. Ferone and F. Murat, Quasilinear problems having natural growth in the gradient: an existence result when the source term is small, in: Équations aux dérivées partielles et applications, Articles dédiés à Jacques-Louis Lions, Gauthier-Villars, Paris, 1998, pp. 497-515.

[15] V. Ferone and F. Murat, Nonlinear problems having natural growth in the gradient: an existence result when the source terms are small, Nonlinear Anal., 42 (2000), 1309-1326.

[16] V. Ferone and F. Murat, Nonlinear elliptic equations with natural growth in the gradient and source terms in Lorentz Spaces, J. Differential Equations 256 (2014), 577-608.

[17] M. Frazier and I. E. Verbitsky, Positive solutions to Schrödinger's equation and the exponential integrability of the balayage, Preprint 2015, arXiv:1509.09005.

[18] M. Fukushima, K. Sato, and S. Taniguchi, On the closable part of pre-Dirichlet forms and the fine support of the underlying measures, Osaka J. Math. 28 (1991) 517-535.

[19] H. A. Hamid and M. F. Bidaut-Veron, On the connection between two quasilinear elliptic problems with source terms of order 0 or 1 . Commun. Contemp. Math. 12 (2010), 727-788.

[20] K. Hansson, V. G. Maz'ya, and I. E. Verbitsky, Criteria of solvability for multidimensional Riccati equations, Ark. Mat. 37 (1999), 87-120.

[21] B. Jaye, V. G. Maz'ya, and I. E. Verbitsky, Existence and regularity of positive solutions of elliptic equations of Schrdinger type, J. Anal. Math. 118 (2012), 577-621.

[22] B. Jaye, V. G. Maz'ya, and I. E. Verbitsky, Quasilinear elliptic equations and weighted Sobolev-Poincaré inequalities with distributional weights, Adv. Math. 232 (2013), 513-542.

[23] M. Kardar, G. Parisi, and Y.-C. Zhang, Dynamic scaling of growing interfaces, Phys. Rev. Lett. 56 (1986) 889-892.

[24] J. Krug and H. Spohn, Universality classes for deterministic surface growth, Phys. Rev. A (3) 38 (1988) 4271-4283.

[25] J. L. Lewis, Uniformly fat sets, Trans. Amer. Math. Soc. 308 (1988), 177-196.

[26] J.-L. Lions, Quelques méthodes de résolution des problèmes aux limites non linéaires, Dunod; GauthierVillars, Paris 1969. xx+554 pp. 
[27] J. Malý and W. P. Ziemer, Fine regularity of solutions of elliptic partial differential equations, Mathematical Surveys and Monographs, 51. American Mathematical Society, Providence, RI, 1997. xiv+291 pp.

[28] V. G. Maz'ya, Sobolev Spaces with Applications to Elliptic Partial Differential Equations, second, revised and augmented ed., in: Grundlehren der math. Wissenschaften, vol. 342, Springer, Heidelberg, 2011.

[29] T. Mengesha and N. C. Phuc Quasilinear Ricatti type equations with distributional data in Morrey space framework, J. Differential Equations 260 (2016), 5421-5449.

[30] P. Mikkonen, On the Wolff potential and quasilinear elliptic equations involving measures, Ann. Acad. Sci. Fenn., Ser AI, Math. Dissert. 104 1996, 1-71.

[31] C. Pérez, Two weighted inequalities for potential and fractional type maximal operators, Indiana Univ. Math. J. 43 (1994), 663-683.

[32] E. T. Sawyer and R. L. Wheeden, Weighted inequalities for fractional integrals on Euclidean and homogeneous spaces, Amer. J. Math. 114 (1992), 813-874.

${ }^{1}$ Department of Mathematical Sciences, Seoul National University, GwanAkRo 1, GwanakGu, Seoul 08826, South Korea.

E-mail address: kadimurthi@snu.ac.kr and karthikaditi@gmail.com

2 Department of Mathematics, Louisiana State University, 303 Lockett Hall, Baton Rouge, LA 70803, USA.

E-mail address: pcnguyen@math.1su.edu 